\title{
Recent advances in cytochrome $c$ biosensing technologies
}

\author{
Pandiaraj Manickam ${ }^{1 *}$, Ajeet Kaushik ${ }^{2}$, Chandran Karunakaran ${ }^{3}$, Shekhar Bhansali ${ }^{1}$ \\ ${ }^{1}$ Bio-MEMS and Microsystems Laboratory, Department of Electrical and Computer Engineering, Florida \\ International University, Miami, FL, USA \\ ${ }^{2}$ Center for Personalized Nanomedicine, Institute of Neuro immune Pharmacology, Department of \\ Immunology, Herbert Wertheim College of Medicine, Florida International University, Miami, FL, USA \\ ${ }^{3}$ Biomedical Research Laboratory, Department of Chemistry, VHNSN College (Autonomous), \\ Virudhunagar, amil Nadu, India
}

*Corresponding Author: Ph. +1 (305)-348-2863. Email: pmanicka@ fiu.edu (P.M.),

\begin{abstract}
This review is an attempt, for the first time, to describe advancements in sensing technology for cytochrome $c$ (cyt $c$ ) detection, at point-of-care (POC) application. Cyt $c$, a heme containing metalloprotein is located in the intermembrane space of mitochondria and released into bloodstream during pathological conditions. The release of cyt $c$ from mitochondria is a key initiative step in the activation of cell death pathways. Circulating cyt $c$ levels represents a novel in-vivo marker of mitochondrial injury after resuscitation from heart failure and chemotherapy. Thus, cyt $c$ detection is not only serving as an apoptosis biomarker, but also is of great importance to understand certain diseases at cellular level. Various existing techniques such as enzyme-linked immunosorbent assays (ELISA), Western blot, high performance liquid chromatography (HPLC), spectrophotometry and flow cytometry have been used to estimate cyt $c$. However, the implementation of these techniques at POC application is limited due to longer analysis time, expensive instruments and expertise needed for operation. To overcome these challenges, significant efforts are being made to develop electrochemical biosensing technologies for fast, accurate, selective, and sensitive detection of cyt $c$. Presented review describes the cutting edge technologies available in the laboratories to detect cyt $c$. The recent advancements in designing and development of electrochemical cyt $c$ biosensors for the quantification of cyt $c$ are also discussed. This review also highlights the POC cyt $c$ biosensors developed recently, that would prove of interest to biologist and therapist to get real time informatics needed to evaluate death process, diseases progression, therapeutics and processes related with mitochondrial injury.
\end{abstract}

Key words: Cytochrome $c$; Point-of-care; Apoptosis; Cancer therapy; Biosensors; Enzymes; Antibodies; Aptamers; 


\section{Introduction}

\subsection{Cytochrome $c$, a brief understanding}

Cytochrome $c$ (cyt $c$ ), a small (molecular weight: 12,500 Da, 104 amino acids), highly water-soluble redox active metalloprotein is located in the intermembrane space of mitochondria (Hayashi and Capaldi, 1972; Martinou et al., 2000). It has a highly conserved three dimensional (3D) structure and a covalently attached active heme prosthetic group (Fig. 1). In mitochondrial respiratory chain of energy production, cyt $c$ functions as a single electron carrier between two membrane bound complexes viz. complex III and (b $c_{1}$ complex or cytochrome $c$ reductase) and complex IV (cytochrome $c$ oxidase). During the mitochondrial electron transfer reaction, the heme active site of cyt $c$ alternates between a reduced ferrous $\left(\mathrm{Fe}^{2+}\right)$ and oxidized ferric $\left(\mathrm{Fe}^{3+}\right)$ states (Mathews, 1985; Skulachev, 1998; Wang et al., 2002). Cyt $c$ is a multi-functional enzyme, involving in both life and death decisions of cell. It participates in electron transfer as a part of the mitochondrial electron transport chain (ETC) and is thus an indispensable part of the energy production process. Its release from mitochondria is an essential step for the formation of the apoptosome and the progression of cell death processes (Hüttemann et al., 2011; Wang, 2001).

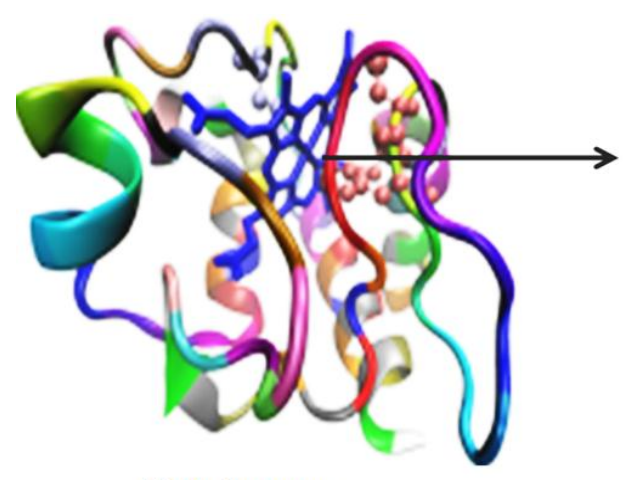

Cytochrome $c$

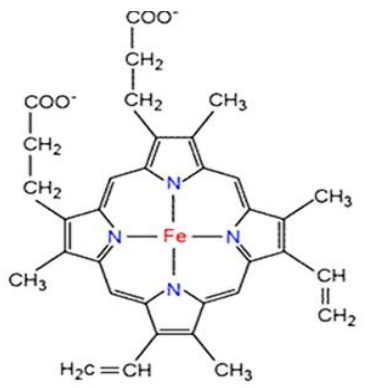

Heme $c$

Figure 1. Cyt $c$ contains one heme prosthetic group inserted into a hydrophobic cleft in the protein.

\subsection{Role of cyt $c$ in mitochondrial electron transport chain}

Mitochondria are often referred to as "powerhouse of the cell", because they produce approximately $90 \%$ of the required energy in the form of adenosine triphosphate (ATP) through oxidative phosphorylation. Thus, the primary role of oxidative phosphorylation is the production of energy, which drives all cellular processes. 


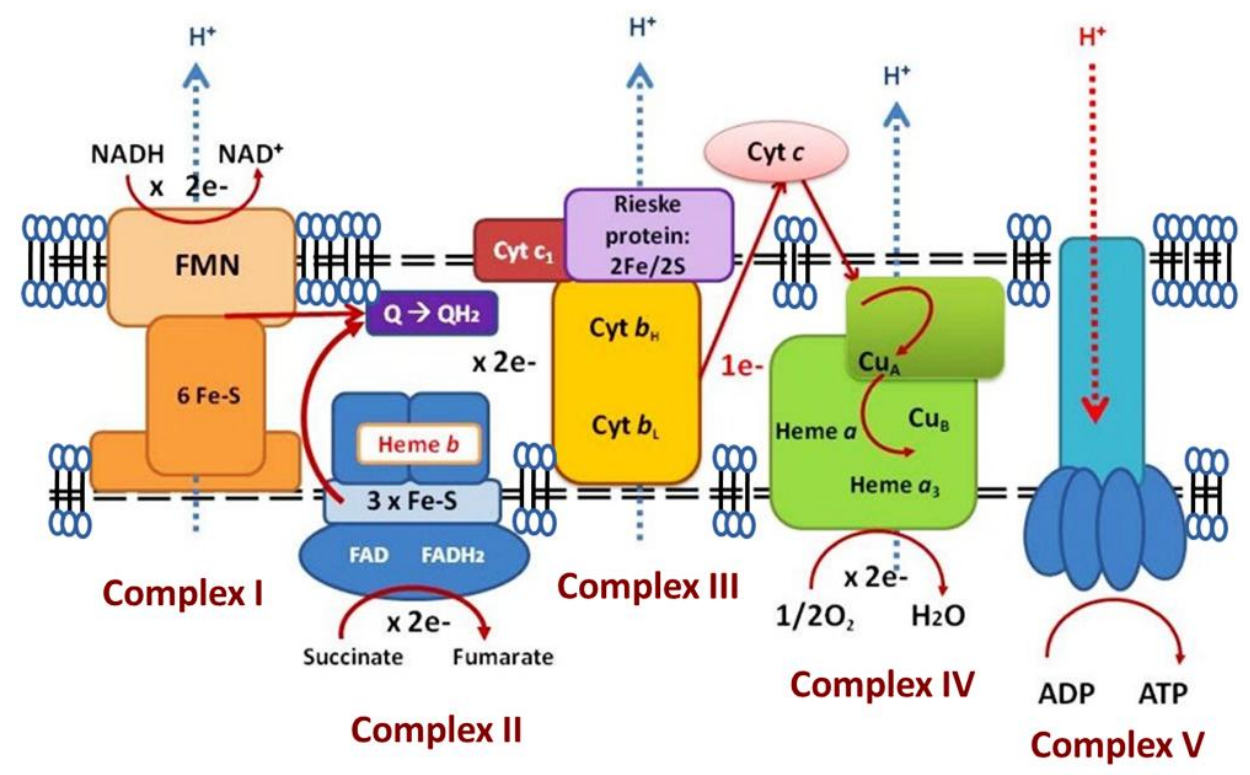

Figure 2.The mitochondrial respiratory chain and oxidative phosphorylation.

Mitochondrial ATP production occurs through the flow of electrons from nicotinamide adenine dinucleotide (NAD) or flavin adenine dinucleotide (FAD) reducing equivalents. These electrons are passed through a series of respiratory complexes in the inner mitochondrial membrane called electron transport system (Fig. 2). It results in the formation of an electrochemical gradient, which enables the ATP synthase to synthesize the energy rich ATP. The mitochondrial respiratory chain comprises series of five multi-subunit enzyme complexes (complexes I, II, III, IV and V) and two electron carriers (ubiquinone and cyt $c$ ). These two electron carriers play critical role in the efficient transfer of electrons in the electron transport chain (Hatefi, 1985; Koopman et al., 2007; Kushnareva et al., 2002).

\subsection{Role of cyt $c$ in apoptosis}

In addition to its central role as power source, mitochondrial respiratory chain is also the major source for generation of reactive oxygen species (ROS) (Kannan and Jain, 2000). Under normal physiological conditions, $1-2 \%$ of molecular oxygen consumed by mammalian cells is metabolized to ROS. Consequently, the ROS including superoxide anion radical $\left(\mathrm{O}_{2}{ }^{-}\right)$, hydrogen peroxide $\left(\mathrm{H}_{2} \mathrm{O}_{2}\right)$, and hydroxyl radicals $\left(\mathrm{OH}^{-}\right)$are constantly formed in all aerobic cells. Low levels of endogenous ROS are essential to the cells for regulating various physiological 
processes such as cell signaling pathways and regular cell proliferations (Burdon et al., 1989). However, an excess production of ROS in cells under various pathological conditions can cause mitochondrial dysfunctions, protein oxidation, DNA mutations and excessive cellular damage, all of which can lead to translocation of cyt $c$ from mitochondria to cytosol. The translocation of cyt $c$ from mitochondria to cytosol is a critical event in the activation of intracellular signaling; it results in a cascade of caspase activation and leads to programmed cell death-apoptosis. The mitochondrial/DNA damage and apoptosis have been implicated in disease that are linked to oxidative stress/hypoxia such as atherosclerosis, cancer, diabetics, rheumatoid arthritis, postischemic perfusion injury, myocardial infarction, cardiovascular diseases, chronic inflammation, stroke, septic shock, aging and other degenerative diseases in humans (Karunakaran et al., 2014; Uttara et al., 2009). Apoptosis or programmed cell death is a regular, highly conserved physiological process, and is an active research topic of biochemical and biomedical sciences. This regulated process is responsible for removal of damaged or infected cells from the cellular population, which links apoptosis to the cell cycle, replication and DNA repair (Kim et al., 2005). Moreover, apoptosis is one of the main mechanisms governing accurate embryonic development and the maintenance of tissue homeostasis. Apoptosis in cells, usually characterized by specific morphological and biochemical changes, viz. nuclear shrinkage, chromatin condensation, DNA fragmentation and membrane blebbing (Culmsee and Landshamer, 2006). The research communities all over the globe predicts that there are two main pathways are involved in apoptotic cell death: (i) the extrinsic or death receptor pathway and (ii) the intrinsic or mitochondrial pathway (Patel et al., 1996; Tait and Green, 2010). In both pathways, translocation of cyt $c$ from mitochondria to cytosol is one of the most important regulatory steps. Once cyt $c$ has been released into the cytosol it interacts with a protein called Apaf-1. This leads to the recruitment of pro-caspase 9 into a multi-protein complex with cyt $c$ and Apaf-1 called the apoptosome. Formation of the apoptosome leads to activation of caspase cascades, which further leads to the activation of apoptosis. Such cyt $c$ release has been reported for apoptosis induced by chemotherapeutic drugs, oxidative stress, UV irradiation, serum, and glucose deprivation. 


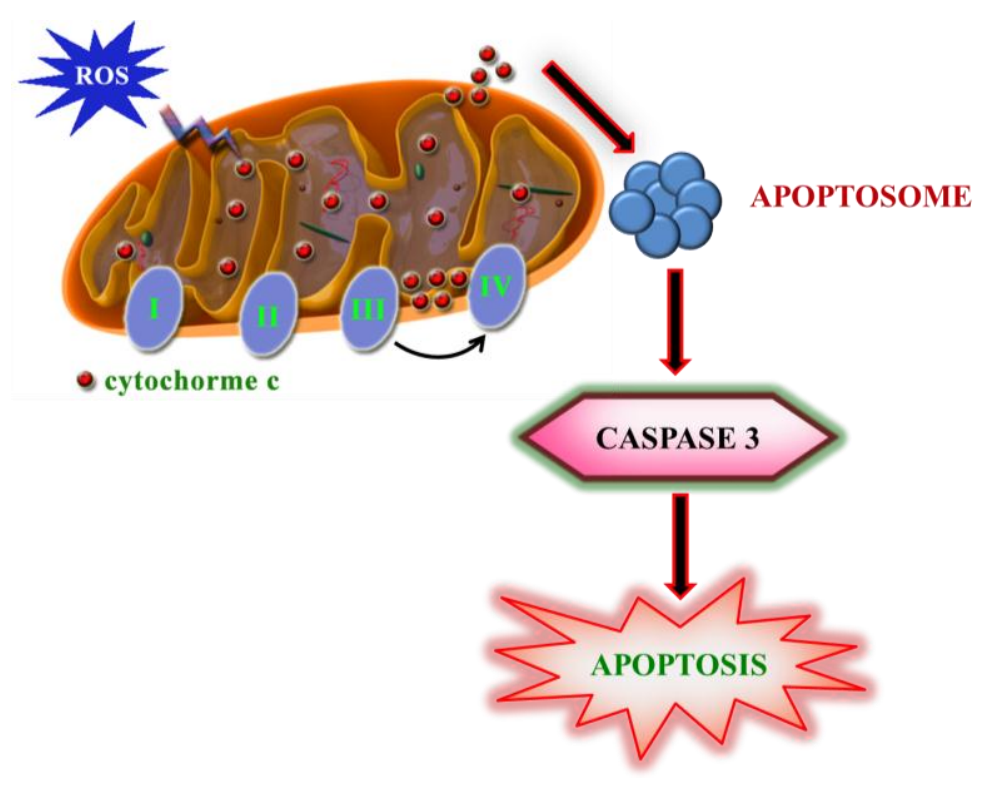

Figure 3. Mitochondrial-mediated apoptotic signaling. Cyt $c$ release from the mitochondrion leads to formation of the apoptosome and activation of procaspase-9. Active caspase- 9 cleaves and activates caspase-3, which leads to apoptosis.

\subsection{Other important biological functions of cyt $c$}

Current cancer therapy protocols such as chemotherapy, $\gamma$-irradiation, or immunotherapy, primarily exerts their anti-tumor effects by inducing apoptosis in cancer cells. This releases the functional apoptosis initiating protein, cyt $c$ into the cytoplasm of cells. Recent reports suggests that cyt $c$ not only released into cytoplasm of cells, but furthermore it leaves the cells and reaches the serum of patients those who undergoes cancer therapy. Thus, the release of cyt $c$ can be used to monitor and evaluate the efficacy of therapy towards cancer treatment.

In the last few decades, it is confirmed that cyt $c$ is also released into the blood circulation, following myocardial infarctions, cardiac arrest and resuscitation. In myocardial infarctions, the oxygen supply to regions of the heart becomes interrupted. During such events cells die, release cyt $c$ into the circulating blood. Recent studies suggest that measuring levels of circulating cyt $c$ could also serve as in vivo marker of mitochondrial injury, organ damage and prognosticate survival after resuscitation from cardiac arrest. Some similar observations have been made in patients with a wide variety of critical conditions. For example, Adachi et al. reported rapid rise in serum cyt $c$ concentrations in patients showing systemic inflammatory response syndrome and multi organ dysfunction syndrome, with higher levels observed in patients who did not survive the episode. Likewise, the levels of cyt $c$ inpatients presenting with 
influenza-associated encephalopathy were significantly higher. Similarly, the study by Sakaida et al., indicated that the assay of serum cyt $c$ is very sensitive for the detection of liver damage, and such assays may also be applicable for prognosis of patients with oxidative, in those including the prediction of prognosis the complication of brain edema (Sakaida et al., 2005). Decrease in the cyt $c$ redox activity was also successfully applied for the sensitive detection of antirespiratory poisons (Fuku et al., 2012). Consequently, the detection of cyt $c$ release is increasingly important as this not only provides valuable information about the nature and extent of apoptosis but serves as a preclinical indicator of various pathologies, medical diagnostics and therapeutic treatment. There is an urgent need in the development of simple and reliable technologies for the detection of cyt $c$. A summary of multi-functional biological role and clinical importance of cyt $c$ is presented in Figure 4.

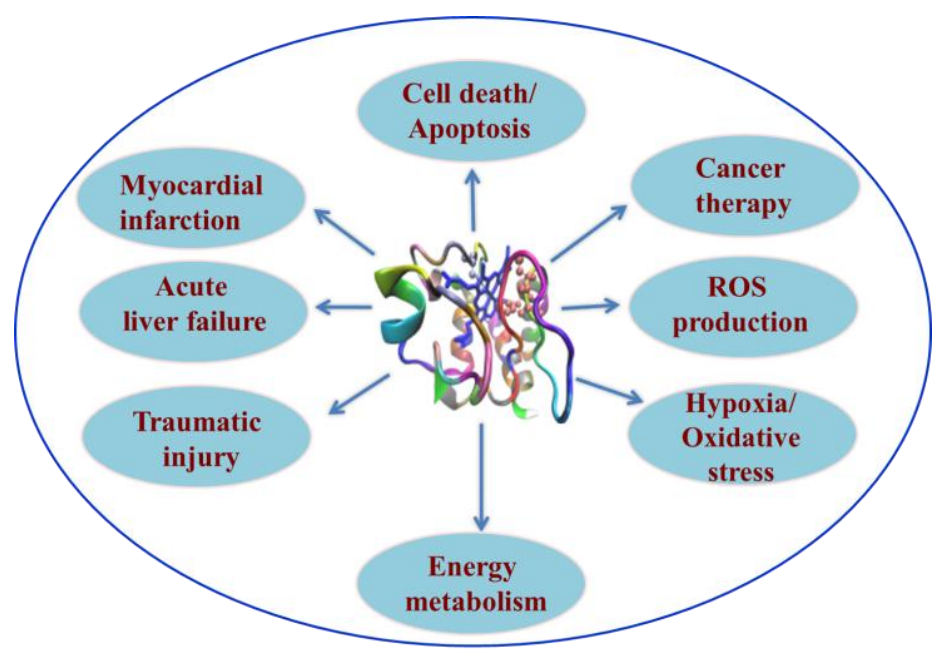

Figure 4. Summary of multiple biological functions and clinical importance of cyt $c$

\section{State-of-the-art of cyt $c$ detection}

\subsection{Flow cytometry}

The techniques available currently at laboratories to measure cyt $c$ release includes flow cytometry, Western blot, and enzyme-linked immunosorbent assay (ELISA). Flow cytometry is a technique for counting, examining and sorting microscopic particles suspended in a stream of fluid. It allows simultaneous multi-parametric analysis, physical and/or chemical characteristics of individual cells within a heterogeneous population of cells. It is an automatic, fast, and reproducible process for the detection and quantification of proteins and biomolecules in cell 
culture. Several authors have demonstrated the detection of cytosolic cyt $c$ released from mitochondria using flow cytometry(Campos et al., 2006; Ng et al., 2012)(King et al., 2007). Although flow cytometry technique is sensitive and can be used to detect early stages of apoptosis(Waterhouse and Trapani, 2003a), this technique however, requires procurement of expensive capital instrument which hinders its use for routine clinical diagnosis. Furthermore, this technique requires a highly trained technician to ensure reliable results.

\subsection{Western blot}

Western blotting (immunoblotting or protein blotting) is one of the important technique for the immunodetection of cyt $c$ (Liu et al., 2012). It involves an electrophoretic sieving (in which proteins are separated by size) followed by an immunoassay. In this technique, first, a mixture of proteins is separated based on molecular weight through gel electrophoresis. These results are then transferred to a membrane producing a band for each protein. The membrane is then incubated with labels antibodies specific to the protein of interest. Western blot technique is routinely implemented for basic research and as a confirmative test for cyt $c$ detection in biological assays and clinical regulatory tests because of high selectivity conferred by using both separation and immunoassay. For example, lung mitochondria and cytosol cyt $c$ protein expression were analyzed using Western blotting technique by $\mathrm{Du}$ et al., in a examining the regulatory effect of $\mathrm{H}_{2} \mathrm{~S}$ on lipopolysaccharide (LPS) induced apoptosis. Walker et al., employed Western blot technique to evaluate the applicability of arsenic trioxide as a potential drug for lung cancer treatment by monitoring cyt $c$ release(Walker et al., 2016). Kavathia et al., developed a Western blotting assay to measure total cyt $c$ in serum from a large clinically defined normal group in order to assess the global balance of systemic markers of apoptosis (Kavathia et al., 2009). Wang et al., investigated the cardioprotective effects of berberine against

myocardial ischemia/reperfusion injury through attenuating mitochondrial dysfunction and myocardial apoptosis. They measured cyt $c$ expression both in mitochondria and cytosol using western blotting (Wang et al., 2015). Although, useful in deriving accurate results, Western blot technique, still suffers from laborious protocols and time consuming procedures that include electrophoresis of the proteins through a gel and transfer to a membrane, membrane blockage, and incubation with primary, secondary antibodies to detect cyt $c$. The total western blot procedure usually takes at least 48 hours to complete and the results are not very accurate as the 
bands obtained with western blot are very difficult to quantify. Moreover, the difficulties in miniaturization and low sensitivity makes the Western blot techniques not suitable for POC diagnosis.

\subsection{ELISA}

The ELISA is another immunological method; like western blot technique, it also utilizes the antibodies for the detection of cy $c$ ELISA is the most effective approach for specific detection of cyt $c$ and has been used extensively for disease diagnoses and in biochemical research(Langs-Barlow et al., 2015; Skemiene et al., 2013). Of late, there are two different formats for ELISA methods widely used for cyt $c$ detection. Two strategies namely (i) Sandwich immunoassay and (ii) Competitive immunoassay have investigated to develop efficient ELISA assays for Cyt c detection, as described below.

\subsubsection{Sandwich Immunoassay}

The sandwich immunoassay is the most commonly used commercial assay for the detection of cyt $c$. In this type of immunoassay, cyt $c$ is "sandwiched" between two antibodies (primary and labeled secondary antibody).

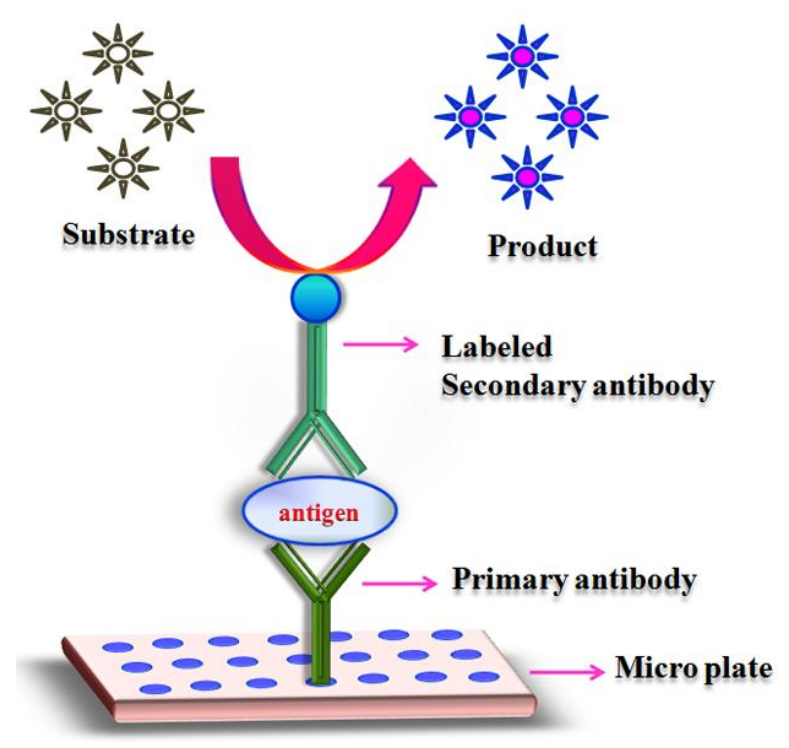

Figure 5. Diagrammatic representation of sandwich ELISA. 
The schematic representation of commonly used protocol adopted for sandwich immunoassay is shown in Figure 5. The first step in the sandwich immunoassay is coating of a constant amount of primary antibody (or capture antibody) specific to cyt $c$ on to the microplate surface. Following coating, addition of a series of dilutions of the cyt $c$ standards to antibodies immobilized on the plate in the next step. After removing unbound cyt $c$ by washings, addition of, an enzyme-linked secondary antibody specific for cyt $c$ is the next step. Following washings to remove any unbound enzyme labeled secondary antibody, a substrate solution usually added to wells and the color developed in proportion to the amount of cyt $c$ bound to the antibody is monitored. The color development will be stopped using a stop solution and the intensity of the color is measured(Dixit et al., 2012). In sandwich type immunoassay, the colorimetric signal produced becomes more intense as the concentration of cyt $c$ in the sample increases.

\subsubsection{Competitive Immunoassay}

Unlike to sandwich type immunoassays, competitive immunoassays for cyt $c$ uses only a single antibody, however it uses a labeled cyt $c$, which competes with unlabeled cyt c to bind with the coated antibody. Direct competitive immunoassays (Fig. 6) involve the immobilization of specific antibody to a microtiter plate. Unlabeled antigen and enzyme-labeled antigen (labeled with HRP or ALP) are competing for the antibody binding sites. The amount of antibody-bound labeled antigen is quantified colorimetrically, where the color intensity is inversely proportional to the amount of free antigen or un-labeled antigen in the sample.

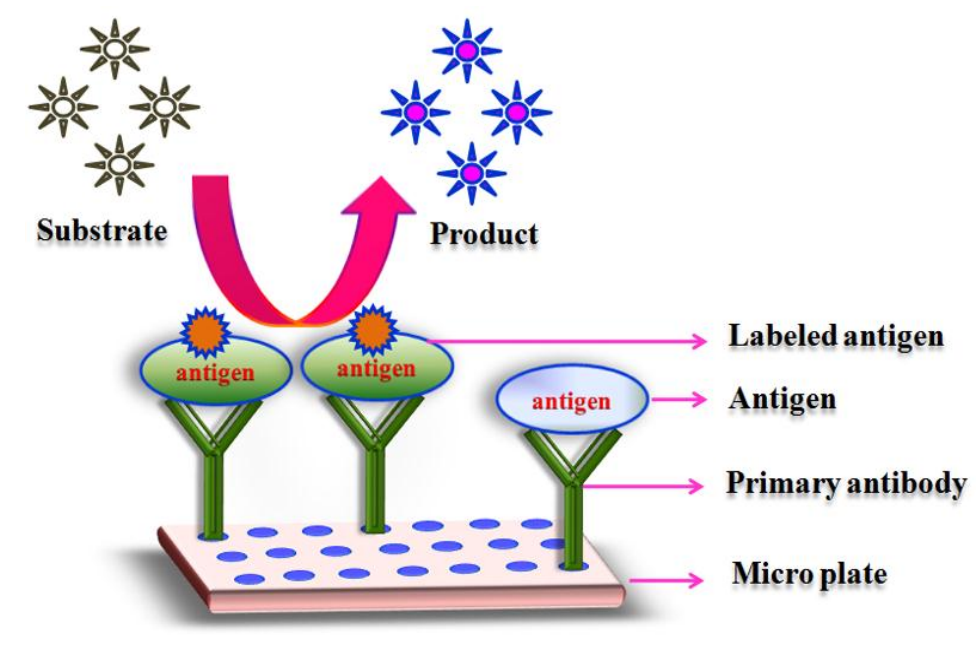

Figure 6. Diagrammatic representation of competitive ELISA. 
In a study by Javid et al., ELISA was used to monitor cyt $c$ levels in serum of cancer patients undergoing chemotherapy(Javid et al., 2015). Similarly, Kadam et al., used ELISA technique to evaluate clinical significance and the changes in concentration of cyt $c$ and other components released in serum of breast cancer patients undergoing adjuvant chemotherapy(Kadam and Abhang, 2015). Barczyk et al., modified commercially available cyt $c$ ELISA to monitor cyt $c$ levels in the extracellular medium of apoptotic cells and in the serum of cancer patients using ELISA, reflecting therapy-induced cell death burden. To increase the sensitivity and the reliability of the ELISA, the group replaced the primary (coating) antibody with a more suitable one(Barczyk et al., 2005). In a study by Jemmerson and coworkers, commercially-available sandwich ELISA and Western blot technique were used to quantify cyt $c$ release in the extracellular fluid from death-induced cultures of cell lines (Jemmerson et al., 2002). Although the commercial test kits provides high sensitivity and selectivity, they usually involves time consuming protocols, require test expensive test kits, bulky ELISA readers, and the demand for skilled professionals. This restricts the deployment of ELISA at POC. Devices for POC cyt $c$ detection must not only be sensitive, but also fulfill the following criteria, of robustness, operation simplicity, and overall cost per assay. To be used for clinical diagnosis, the devices must be accurate enough to detect normal as well as elevated levels of the protein concentrations and resistant to false positive and false negative alarms. To design sensitive and cost-effective medical technologies that diagnose clinical abnormalities, researchers all over the globe paying close attention to personnel health-care monitoring systems with the use of biosensors.

\subsection{Fluorescent activation image analysis}

The release of cyt $c$ from mitochondria to cytosol can also be visualized with an aid of fluorescence imaging tools. Tagging of cyt $c$ with green fluorescent protein (GFP)-tagged cyt $c$ has made it possible to tracking cyt $c$ redistribution within cells in real time. For example, Waterhouse studied the translocation of cyt $c$ from mitochondria by selective permeabilization of the plasma membrane followed by immunocytochemistry and fluorescence microscopy. This assay is based on the idea that permeabilization of cells will allow cytoplasmic cyt $c$-GFP to diffuse out of the cells. Cells with cytoplasmic cyt $c$-GFP will therefore have less GFP fluorescence than cells with intact mitochondria. This method, however, is only useful for cells 
that express GFP-cyt $c$ (Waterhouse and Trapani, 2003b). Similarly, Goldstein et al., studied the mechanism and kinetics of cyt $c$ by using GFP tagged cyt $c$ (Green et al., 2000). In another study, the research group utilized a cyt $c$ fusion that binds fluorescent biarsenical ligands (cyt $c$-4CYS) as well as cyt $c$-GFP to track cyt $c$ translocation from mitochondria in different cell types during apoptosis (Goldstein et al., 2005). Chen et al., reported aptamer based nanosensor that realizes the direct fluorescence activation imaging of cyt $c$ translocation from mitochondria(Chen et al., 2015). This strategy relies on spatially selective cytosolic delivery of a nanosensor constructed by assembly of a fluorophore-tagged DNA aptamer on PEGylated graphene nanosheets. The cytosolic release of cyt $c$ is able to dissociate the aptamer from graphene and trigger an activated fluorescence signal. It also enables real-time visualization of the Cyt c release kinetics and direct identification of the regulators for apoptosis. Ohana et al., used yellow fluorescent protein (YFP) fragments to fuse with cyt $c$ to screen the molecular events that happens during mitochondrial apoptosis (Yivgi-Ohana et al., 2011). The YFP system enables to monitor the conformational changes associated with activation of other apoptotic proteins.

\section{Biosensor for cyt $\boldsymbol{c}$ detection}

A biosensor is an analytical device used for the detection of analytes, consisting of three parts: (i) the bioreceptor, (ii) the transducer or the detector element, and (iii) the reader device. A bioreceptor or biorecognition element is a biomolecule that recognizes the target analyte. The transducer converts the biorecognition event into measurable signal. The reader unit is used to analyze and display the results. Biosensors can be classified based on either (i) biorecognition element used or (ii) the transduction principle employed. Figure 7 depicts working mechanism of biosensor with various recognition elements used in the literature for cyt $c$ detection.

Enzymes, antibodies, and aptamers are the main classes of biorecognition elements widely used in biosensing applications. Biosensors uses enzymes are called catalytic biosensors, where the enzymes catalyzes the conversion of the analyte into a product. On the other hand, biosensors which uses antibodies and aptamers, fall under the category of affinity biosensors. These affinity biosensors can be named as immunosensors, if antibodies are used as recognition element; and called as aptasensor if aptamers are used as recognition elements. Among the great varieties of transduction techniques, electrochemical and optical methodologies have evolved as versatile and powerful techniques for accurate biosensing. The biosensor to be 
applied for POC detection system should fit in to the listed criteria. A) Label-free: The biosensor should be label-free and should not require a labeling process for the detection of target molecules because the labelling process are complicated, costly, and time consuming. B) Selectivity: To avid false positive and false negatives, the receptor should have good selectivity for the target analyte. Selectivity is the most crucial criterion for the reliable operation of a POC system. However, ultra-sensitivity is not required because upon positive diagnosis by the POC system, the analyte should be accurately reanalyzed to determine the exact phase of the disease with cutting edge technologies in a central laboratory. C) Integration and packaging: For an inexpensive POC system, the biosensor should be easily integrated and packaged with userinterface electronics to minimize the system size and production cost.

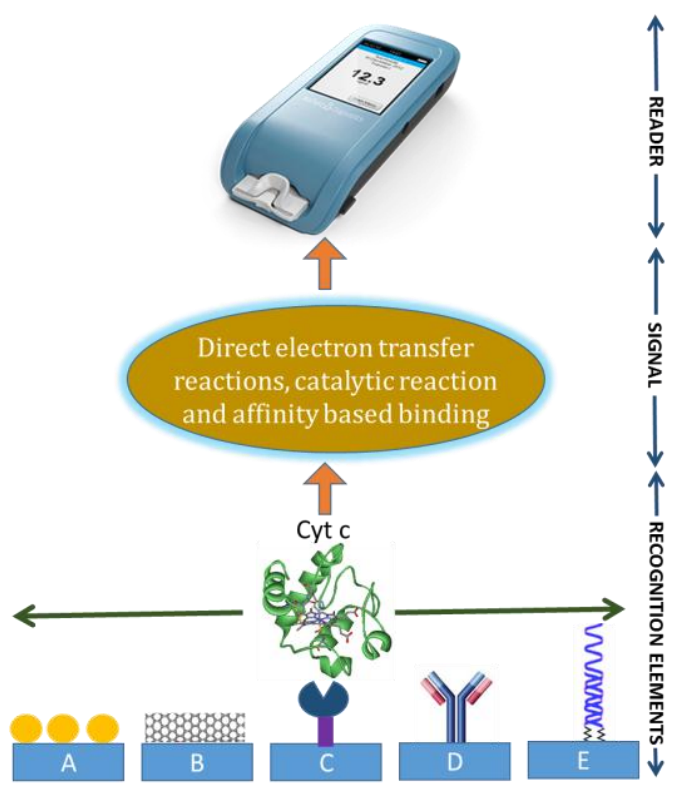

Figure 7. Different types of transducers and detection methodologies used for cyt $c$ sensing. Electrode surfaces modified with (A) AuNPs, (B) CNTs, (C) Enzymes, (D) Antibodies, and (E) Aptamers

\subsection{Enzymatic biosensor for cytochrome c}

Cyt $c$ exists in an oxidized (ferric) or reduced (ferrous) form. Inter-conversion between the two states is brought about by catalytic reaction with two different membrane bound enzymes, namely, cytochrome $c$ oxidase $(\mathrm{CcO})$ and cytochrome $c$ reductase $(\mathrm{CcR})$. $\mathrm{CcO}$ oxidizes reduced form of cyt $c$ while $\mathrm{CcR}$ reduces its oxidized form. Although the structures of the two forms of cyt $c$ are similar, the difference in oxidation state make significant difference in binding 
and biochemical properties. As recently reviewed by Brown and Borutaite, the reduced and oxidized forms of cyt $c$ have different abilities to activate apoptosis(Brown and Borutaite, 2008).

\subsubsection{Cytochrome $c$ oxidase based biosensor}

The $\mathrm{CcO}$ is a complex metallo-enzyme containing 13 protein subunits and four metal centers $\left(\mathrm{Cu}_{\mathrm{A}}\right.$, heme $a$, heme $a_{3}$, and $\left.\mathrm{Cu}_{\mathrm{B}}\right)$ with a combined molecular weight of $\sim 2,05,000 \mathrm{Da}$ for the monomeric enzyme(Kadenbach and Hüttemann, 2015; Michel, n.d.) Being the terminal complex (complex IV) of ETC, $\mathrm{CcO}$ accepts electrons from reduced cyt $c$ and transfers them to molecular oxygen. This reaction is coupled to the transfer of protons from the matrix to the intermembrane space by $\mathrm{CcO}$. Although it has 13 subunits, the subunits I and II are essential for the function of enzyme. Subunit I has three of the four metal catalytic sites: heme $a$, and the heme $a_{3} / \mathrm{Cu}_{\mathrm{B}}$ center, where the oxygen reduction takes place, a process which is coupled to proton translocation. Four electrons from cyt $c$ (one from each cyt $c$ molecule) are subsequently transferred via the $\mathrm{Cu}_{\mathrm{A}}$ center in subunit II and heme $a$ to the oxygen reduction center heme $a_{3} / \mathrm{Cu}_{\mathrm{B}}$ (Fig. 8).

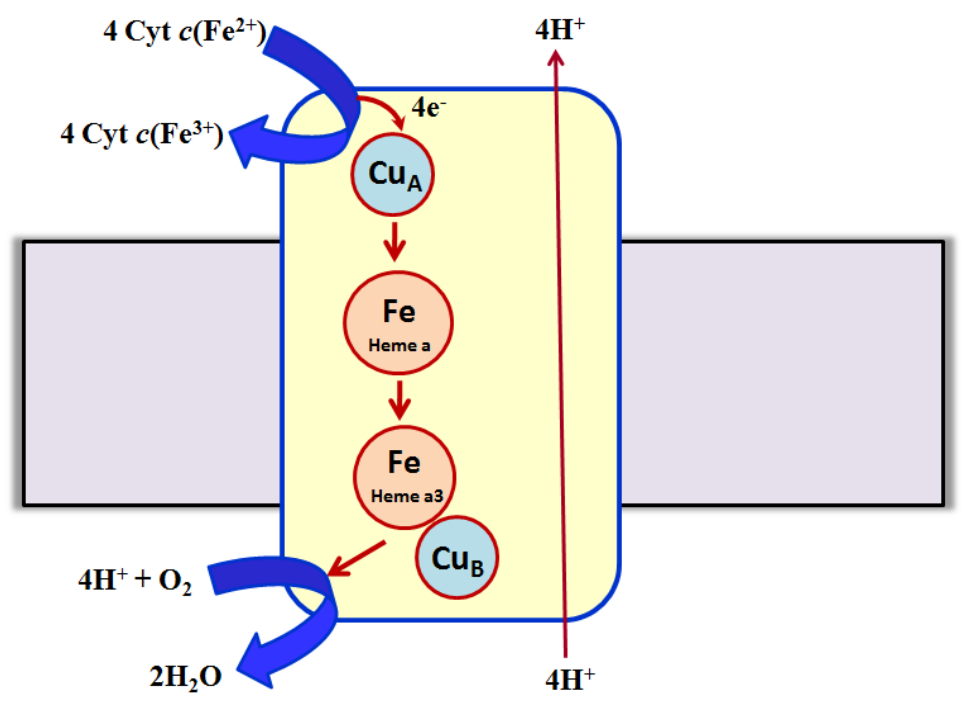

Figure 8. Schematic representation of electron transport through various active sites of mitochondrial complex IV $(\mathrm{CcO})$.

Interesting feature of mitochondrial membrane proteins, especially $\mathrm{CcO}$ are its high hydrophobicity and low stability, which makes them very difficult to immobilize on the electrode surface, consequently, use in biosensor applications. Still, a number of biochemical and biophysical studies have challenged the immobilization of $\mathrm{CcO}$ on the electrode surface by 
coating the surface with lipid bilayers. Such bilayer coatings are considered as simple models for biological membranes, mimicking the natural environment of the integral membrane proteins which provide improved stability. The environment maintains the structural and functional integrity of membrane proteins at the electrode surface (Burgess et al., 1998; Cullison et al., 1994; Salamon and Tollin, 1996). The dimensions of these bilayer structures employed were approximately $50 \AA$ (Rhoten et al., 2002; Tsukihara et al., 1995). Hawkridge and his team has developed an approach that couples thiol self-assembly with deoxycholate dialysis, to create an electrode-supported lipid bilayer membranes for the immobilization of $\mathrm{CcO}$ (Cullison et al., 1994; Rhoten et al., 2002). A submonolayer of octadecyl mercaptan serves to anchor the bilayer on the electrode surface. This group also demonstrated the potentiometric and amperometric biosensing of reduced form of cyt $c$ using the $\mathrm{CcO}$ immobilized electrodes (Lewis et al., 2006). The biosensor showed a linear relationship for the cyt $c$ concentration range from $1 \mu \mathrm{M}$ to 10 $\mu \mathrm{M}$ with a detection limit of $0.1 \mu \mathrm{M}$ at a signal to noise ratio of 5 . In an another biosensing application, the research group demonstrated the effect of temperature and $\mathrm{pH}$ on the effect of $\mathrm{CcO}$ immobilization towards cyt $c$ detection (Rhoten et al., 2000). Octadecanethiolate submonolayer was derivatized on the surface of gold quartz crystal microbalance electrode to anchor the bilayer and immobilize $\mathrm{CcO}$. The sensor system thus designed, amperometrically detected cyt $c$ at a lowest concentration of $0.25 \mu \mathrm{M}$, with a signal to noise ratio of approximately 2.5.

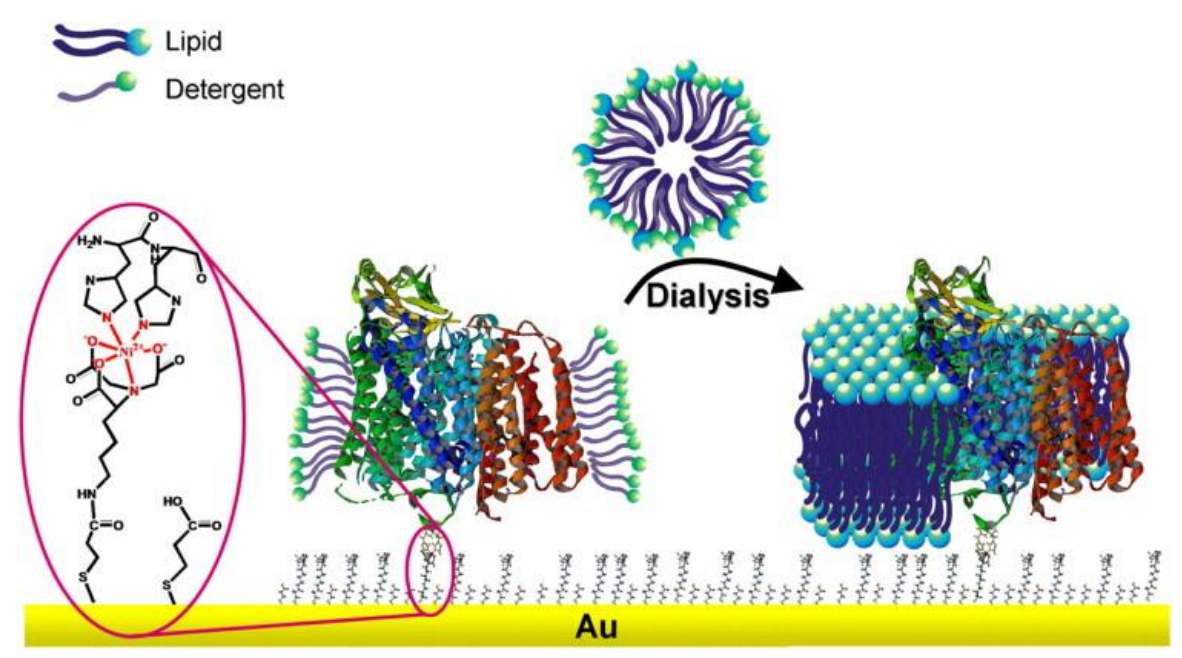

Figure 9. Schematic representation of immobilization of $\mathrm{CcO}$ onto a protein-tethered bilayer lipid membrane (Friedrich et al., 2008) (reproduced with permission from Elsevier Limited). 
Similar biomimetic systems involving histidine (his)-tag technology was also demonstrated for the immobilization of $\mathrm{CcO}$. Friedrich et al., attempted to preserve the catalytic activity of $\mathrm{CcO}$ on a gold film electrode by reconstituting the enzyme with a protein-tethered bilayer lipid membrane (Friedrich et al., 2008). However, in this orientation, the cyt $c$ binding site is directed away from the electrode pointing to the outer side of the protein-tethered bilayer lipid membrane architecture (Fig. 9). Ashe et al., have designed a $\mathrm{CcO}$ based biosensor for the detection of cyt $c$ levels in serum (Ashe et al., 2007). The biosensor was constructed by immobilizing the enzyme, $\mathrm{CcO}$ in didodecyldimethylammonium bromide (DDAB) lipid vesicle dispersion on to a gold electrode. The DDAB vesicles in aqueous solution mimic the environment of inner mitochondrial membrane and provide suitable platform for the effective immobilization of $\mathrm{CcO}$. The vesicles also enables the direct electron transfer between the electrode and the enzyme's redox centres without using any mediators. Square-wave voltammetry was used to detect cyt $c$ levels in human serum samples. The sensor's response to cyt $c$ was linear from $0.2 \mu \mathrm{M}$ to $4 \mu \mathrm{M}$. The lowest level of cyt $c$ detected by this sensor $(0.2 \mu \mathrm{M})$, was much lower than the lowest level of $0.8 \mu \mathrm{M}$ reported previously for patients with myocardial infarctions.

Covalent immobilization of $\mathrm{CcO}$ on the electrode surface has also been reported for the design of cyt $c$ biosensor. Thiol based self-assembled monolayer (SAM) to immobilize the $\mathrm{CcO}$ to detect reduced form of cyt $c$ has been reported by few research groups. Li et al., have reported the covalently binding of $\mathrm{CcO}$ with SAM of 3-mercaptopropionaic acid on a gold electrode by using a carbodiimide coupling ( $\mathrm{Li}$ et al., 1996). Immobilized $\mathrm{CcO}$ exhibits a reversible voltammteric response due to the redox reaction of cyt $a_{3}$ in the enzyme active site and mediate the redox reaction of cyt $c$ in the solution. The electron transfer rate constant of the $\mathrm{CcO}$ based biosensor was depends strongly on the strength and composition of the supporting electrolyte. Recently, Batra et al., fabricated an amperometric biosensor for determination of cyt $c$ by immobilizing $\mathrm{CcO}$ onto nickel oxide nanoparticles decorated carboxylated multiwalled carbon nanotubes/polyaniline film electrodeposited gold electrode (Batra et al., 2013). The sensor had a good linear relationship between the current $(\mathrm{mA})$ and cyt $c$ concentration in the range $5 \mathrm{pM}$ to $500 \mathrm{nM}$ with the detection limit was $5 \mathrm{pM}(\mathrm{S} / \mathrm{N}=3)$. Serum cyt $c$ was also estimated for POC applications. 
Although $\mathrm{CcO}$ based biosensors mainly used to measure reduced form of cyt $c$ by oxidizing it, there are some reports showing $\mathrm{CcO}$ could also use to detect oxidized form of cyt $c$. For example, a study by Cullison and Hawkridge showed that $\mathrm{CcO}$ can measure either both oxidized or reduced cyt $c$ when an appropriate voltage is applied (Cullison et al., 1994). However, the rate of reduction of oxidized form of cyt $c$ by $\mathrm{CcO}$ was less than the oxidation of reduced form of cyt $c$. Ashe et al., also used to detect oxidized form of cyt $c$ in serum samples using $\mathrm{CcO}$ to evaluate its possible application in detecting early myocardial infarctions(Ashe et al., 2007).

Recent discovery by Pasdois et al., suggested that the oxidized cyt $c$ plays an important role in triggering the caspase activation and apoptosis induction (Pasdois et al., 2011). Moreover, in permeablized cell models, the cytosolic cyt $c\left(\mathrm{Fe}^{2+}\right)$ is rapidly oxidized to $\mathrm{Fe}^{3+}$ by the mitochondrial $\mathrm{CcO}$ (Brown and Borutaite, 2008)thus making the $\mathrm{CcO}$ based biosensors difficult to quantify the apoptotic form of cyt $c\left(\mathrm{Fe}^{3+}\right)$. In addition, upon immobilization, it is reported that the electron transfer is blocked in active centers of the $\mathrm{CcO}$ (Hrabakova et al., 2006). Since the analytical applications of $\mathrm{CcO}$ based biosensors are limited only to reduced form of cyt $c$, there is a real need for developing assay for measuring oxidized form of cyt $c$. Our group has developed an alternate biosensor for the quantification of oxidized form of cyt $c$ by immobilizing $\mathrm{CcR}$ onto the nanocomposite modified electrodes. $\mathrm{CcR}$ is a complex III of the electron transport system, catalyzing one electron reduction of oxidized cyt $c\left(\mathrm{Fe}^{3+}\right)$.

\subsubsection{Cytochrome $c$ reductase based biosensor}

$\mathrm{CcR}$ (complex $b c_{1}$ ) is also an integral membrane protein of the mitochondrial respiratory chain. The overall reaction catalyzed by $\mathrm{CcR}$ involves the reduction of two molecules of cyt $c$ and the 2-electron oxidation of ubiquinol $\left(\mathrm{QH}_{2}\right)$ to ubiquinone $(\mathrm{Q})$. This process is coupled with transfer of four protons from the mitochondrial matrix to the intermembrane space.

Ubiquinone +2 cyt $c\left(\mathrm{Fe}^{3+}\right)+2 \mathrm{H}^{+}{ }_{\text {in }} \rightarrow$ ubiquinol +2 cyt $c\left(\mathrm{Fe}^{2+}\right)+4 \mathrm{H}_{\text {out }}^{+}$(Eqn 1.1)

Mitochondrial $\mathrm{CcR}$ is a homodimer with 11 polypeptide chains, while prokaryotic $\mathrm{CcR}$ complexes contain as few as 3 polypeptide chains (Millett et al.). The $b c_{1}$ complex contains three protein subunits with redox prosthetic groups known as the core subunits: (i) a di-heme cyt $b$ containing both the relatively high-potential $b_{\mathrm{H}}$ heme and the lower potential $b_{\mathrm{L}}$ heme; ii) cyt $c_{1}$ 
with the $c$-type heme; and (iii) Rieske protein containing an [2Fe-2S] iron-sulfur cluster [22-24]. According to a well-known biochemical knowledge, cyt $c\left(\mathrm{Fe}^{3+}\right)$ acquires electrons from Fe-Scyt $c_{1}$ clusters of $\mathrm{CcR}$ and gets reduced (cyt $c\left(\mathrm{Fe}^{2+}\right)$ ) by following $\mathrm{Q}$ cycle mechanism(Crofts et al., 2008) (Fig. 10). Experimental evidence indicates that the peripheral domain of the Rieske iron protein moves back and forth between positions close to cyt $b$ and cyt $c_{1}$ thus facilitating the electron transfer within the CcR (Kramer et al., 2004).

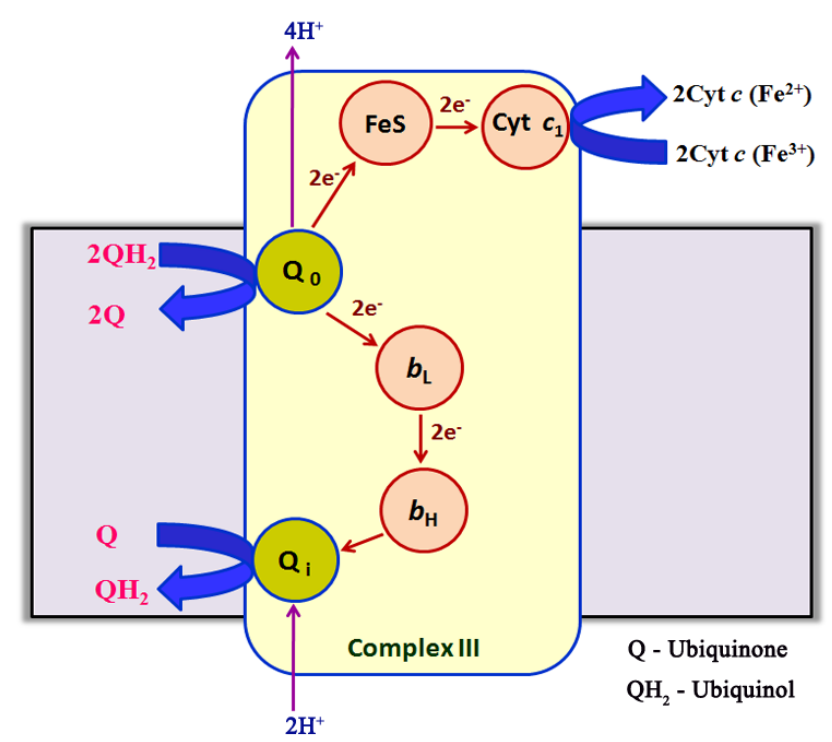

Figure 10. Schematic illustration of electron transport through various active sites of mitochondrial complex III.

Our group reported a novel approach for the detection of oxidized form of cyt $c\left(\mathrm{Fe}^{3+}\right)$ for the first time using CcR immobilized on nanoparticles decorated electrodes (Pandiaraj et al., 2014a, 2013a). Two different kinds of nanomaterial decorated biosensor platforms were used for the construction of biosensors: (a) carbon nanotubes (CNT) incorporated polypyrrole (PPy) matrix on Pt electrode and (b) self-assembled monolayer (SAM) functionalized gold nanoparticles (GNP) in PPy-Pt. The incorporation of CNT/GNP into the PPy matrix exhibited nanoporous structures with large effective surface area for the immobilization of $\mathrm{CcR}$ and enhanced the enzyme biocatalytic activity for the sensitive determination of cyt $c$. The CcR-CNT based biosensor achieved a very low detection limit, wide linear range and high sensitivity over the CcR-GNP based biosensor. The schematic representation of cyt $c$ biosensor architectures were illustrated in Figure 11. Furthermore, the CcR-CNT based cyt $c$ biosensor was applied to 
quantify the cytosolic cyt $c$ released from the mitochondria of apoptotic human lung carcinoma A549 cells and the results were validated with the standard western blot analysis(Pandiaraj et al., 2013b).

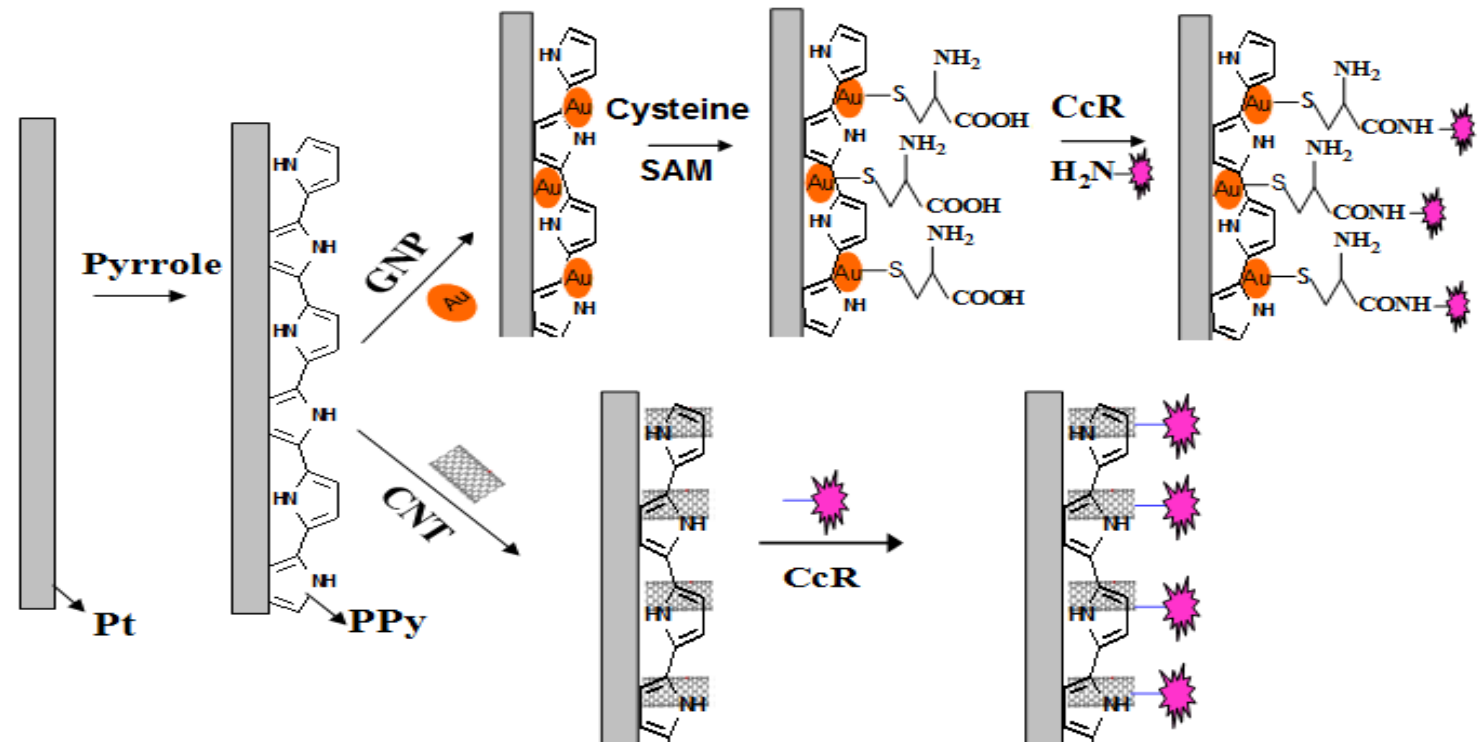

Figure 11. Schematic representation of working mechanism of CcR based biosensors(Pandiaraj et al., 2013b) (reproduced with permission from Elsevier Limited).

Replacement of conventional electrochemical cells by screen printed electrodes (SPE) connected with portable potentiostats is a main trend in the shift of lab electrochemical equipment to hand-held field analyzers. The SPE system can be considered as a disposable electrochemical cell, which reduces the required sample volume, simplifies the apparatus, and makes the point-of-care testing easy to handle and cost effective(Carvajal et al., 2012; Gilbert et al., 2011). In one of the approach for detecting oxidized form of cyt $c$ levels, our group have combined the distinct advantages of CcR functionalized SPE(Pandiaraj et al., 2014a). The developed cyt $c$ assay consists of two parts: (i) a miniaturized electrochemical biosensor based on cytochrome $c$ reductase $(\mathrm{CcR})$ functionalized screen printed electrodes (SPE); (ii) a microcontroller based data acquisition unit integrated with potentiostat circuit capable of performing cyclic voltammetry technique for the analysis. The working electrode surface of SPE was integrated with polypyrrole (PPy)-carbon nanotubes (CNT) nanocomposite for an enhanced immobilization of the enzyme, CcR (Fig. 12). The performance of the volume miniaturized SPE based biosensor coupled with the portable microcontroller based instrument was evaluated, by 
applying it for the measurement of mitochondrial cyt $c$ release during cardiomyocytes apoptosis. The results are validated well with the commercial electrochemical analyzer and standard ELISA.

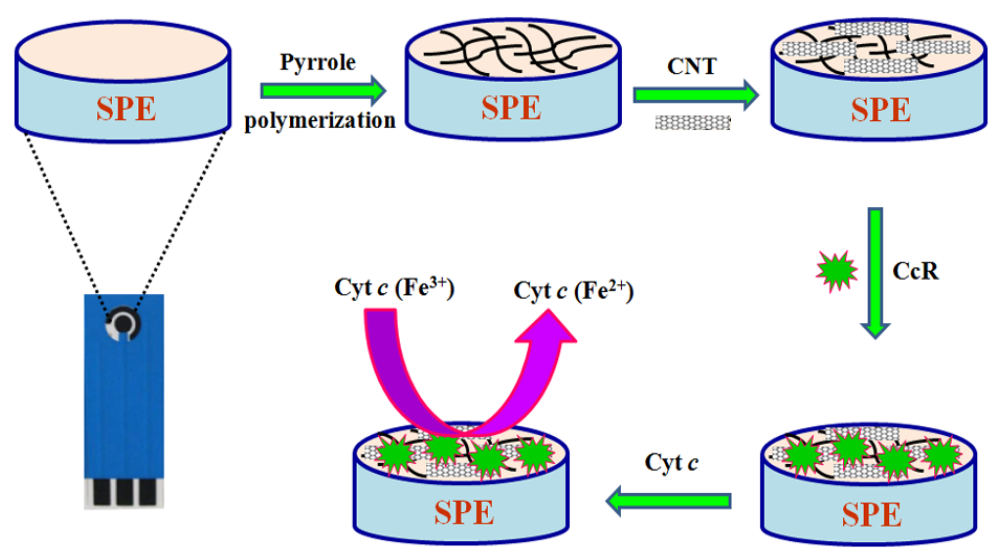

Figure 12. Schematic diagram of the miniaturized cyt $c$ biosensor assay(Pandiaraj et al., 2014a) (reproduced with permission from Elsevier Limited)

\subsection{Cyt $c$ immunosensors}

Immunoassays are quantitative analytical methods that make use of antibodies as immunological reagents. Antibodies are very selective and only bind to their specific targets, even in the presence of a huge range of other materials in a sample. The highly specific binding and large association constants $(\mathrm{Kd})$ of antibodies make them useful for detection and quantitation of analytes in complex sample matrices ranging from culture media to body fluids such as blood, urine, saliva, and sweat. (Li et al., 2008; Pandiaraj et al., 2014a; Rieger et al., 2009). ELISA is an important commercial immunoassay widely employed for protein detection(Lilja et al., 2008; Taufika Islam Williams et al., 2007). However, ELISA is an optical approach, has limitations in colored samples analysis, experimentaltime, and sample size. Hence , it is difficult to employ ELISA for point-of-care testing (vide supra) In this context, the development of electrochemical immunosensors as an alternative to the conventional immunoassay systems, drawing more attention in a wide range of uses, especially for determination of clinically important analytes. Electrochemical immunosensors can provide a precise and real-time measurement of protein biomarkers owing to their unique combination of exquisite specific antigen-antibody interaction and sensitive electrochemical transduction (Guodong Liu et al., 2007; Viswanathan et al., 2009) 


\subsubsection{Labelled immunosensors:}

As the antibody and antigen are intrinsically unable to act as redox partners, most of the immunosensors requires a label that aids in generating an electrochemical signal. The labels are often conjugated to either the antibody (eg. sandwich immunoassay) or antigen (eg., competitive immunoasssay). These labels can be enzymes (ELISA), radio isotopes (radio immunoassay (RIA)), fluorescence probes, or chemiluminescent markers. Among these, ELISA has grown a much interest in the recent years because of its simplicity. The enzymes like horse-radish peroxidase (HRP) and alkaline phosphatase (ALP) are mainly used as the enzymatic labels in ELISA.

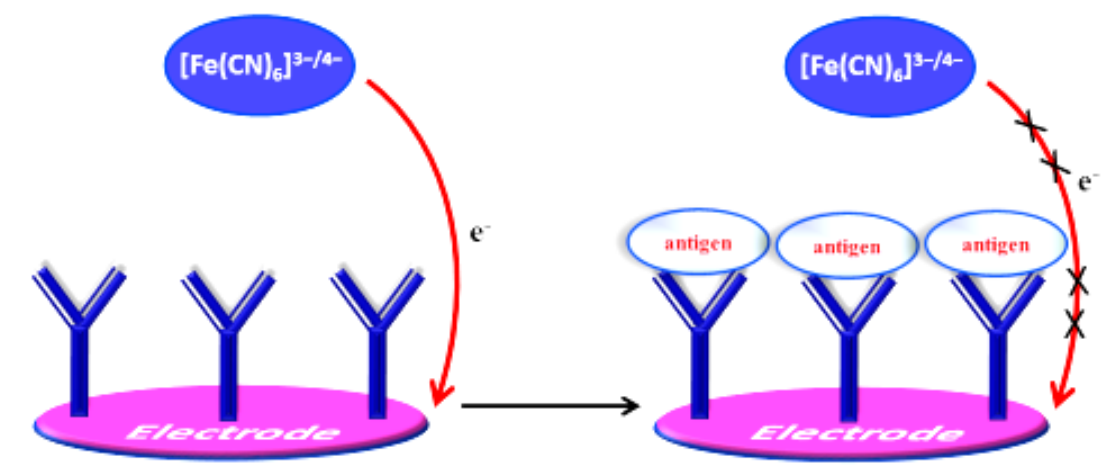

Figure 13. Working principle of label or mediator based immunosensors

Commonly, there are two different formats for ELISA is available for antigen detection: sandwich assays and competitive assays (vide supra). In label-free or direct immunoassays, antibodies are immobilized on the sensor surface plate and subjected to the binding interaction with the antigen of interest. On specific molecular recognition of the antigen by the immobilized capture antibody, there will be changes in the interfacial charge, current, capacitance, impedance, mass and thickness at the immunosensor surface, which in turn has a direct effect on the electron transfer reaction of the probe at the immunosensor-electrolyte solution interface. So, the direct electrochemical immunoassay is usually followed the measurement of impedance, capacitance, current, potential changes due to the formation of immunocomplex. In impedimetric immunosensors, the protein levels could be detected by monitoring the impedance changes caused by immunocomplex with an aid of electrochemical redox probe $\left(\left[\mathrm{Fe}(\mathrm{CN})_{6}\right]^{3-/ 4-}\right)$ (Fig. 13).

\subsubsection{Fluorescent labels}


In fluorescent biosensors, a fluorescent probe attached receptor (antibody or aptamer) binds to the specific protein and transduce a fluorescent signal which can be readily detected and measured. In some cases fluorescence quenching of labels were used for the detection of cyt $c$. Various fluorescent probes including metal nanoclusters, quantum dots. Shamsipur et al., reported two different fluorescent metal nanoclusters labels for the detection cyt $c$ (Shamsipur et al., 2016). They are, (i) hemoglobin-stabilized gold nanoclusters, and (ii) aptamer-stabilized silver nanoclusters. A limit of detection in the nanomolar level (about $15 \mathrm{nM}$ ) was achieved for Cyt $c$ detection using both AuNCs and AgNCs based sensing platforms. Selective quenching effect of glutathione-capped CdTe quantum dots by cyt $c$ has been reported by Cao et al., for cyt $c$ detection(Cao et al., 2009). This method is selective to cyt $c$ as only cyt $c$ quenched the fluorescence of the GSH-CdTe QDs (at $\mathrm{pH}>8.0$,), and no significant fluorescence changes were observed for hemoglobin or other proteins. The linear range of the calibration curve obtained with this method was from $3.2 \times 10^{-8}$ to $2.4 \times 10^{-6} \mathrm{~mol} \mathrm{~L}^{-1}$ and the detection limit is $3.0 \times 10^{-9} \mathrm{~mol} \mathrm{~L}^{-1}$. Yin et al., tagged cyt $c$ binding aptamer with a fluorescent probe called carboxy fluorescein and used vandium sulfide nanosheets to absorb to the probe and quench its fluorescence efficiency(Yin et al., 2015). The proposed sensing system shows gives a linear range of $0.75 \mathrm{nM}$ to $50 \mu \mathrm{M}$, and a limit of detection of $0.50 \mathrm{nM}$.

\subsubsection{Chemiluminescent labels}

Chemiluminescence resulting from the reaction of luminol and oxidants $\left(\mathrm{H}_{2} \mathrm{O}_{2}\right)$ has been extensively studied and applied for the determination of protein biomolecules. For example, Robert Feissner reported a CL procedure for the determination of Cyt $c$ using a variety of luminol-based substrates, giving a linear ranging from 4 to $400 \mathrm{ng}$ (Feissner et al., 2003). Li et al., used a complex of cyt $c$ and $\mathrm{H}_{2} \mathrm{O}_{2}$ to catalyze chemiluminescence reaction of luminol- $\mathrm{H}_{2} \mathrm{O}_{2}$ for cyt $c$ detection. The method showed linearity over the concentration of cyt $c$ ranging from 5 to $700 \mathrm{ng} \mathrm{ml}^{-1}$, with a detection limit of $2 \mathrm{ng} \mathrm{ml}^{-1}(3 \sigma)$ (Li et al., 2010). Dong et al., investigated electrogenerated chemiluminescence behavior of luminol at $\mathrm{CdS}$ quantum dots, graphene, and CdS/graphene (CdS/GR) nanocomposites modified gold electrodes. Inhibition of chemiluminescence intensities of the nanocomposite probe in by cyt $c$ was used for cyt $c$ detection (Dong et al., 2016).

\subsubsection{Electrochemiluminescent labels}


Sakaida et al., proposed a sandwich electrochemiluminescence (ECL) immunoassay serum cyt $c$ level measurement using ruthenium-chelate labeled secondary antibodies(Sakaida et al., 2005). An ECL aptasensor for the detection of cyt $c$ was proposed by Bin et al., using CdS:Mn quantum dot-modified $\mathrm{TiO}_{2}$ nanowires as ECL emitter (Bin et al., 2016). Cyt c can be sensitively detected based on the quenching of ECL with a LOD of $9.5 \mathrm{fM}$. Hu et al., synthesized graphene oxide sheets/polyaniline/CdSe quantum dots nanocomposite for the ECL detection of cyt $c$ (Hu et al., 2013). The detection mechanism is based on the measurement of reduction quenching effect of the nanocomposite when it reacts with cyt $c$. Under the optimized conditions, the ECL intensity decreased linearly with the cyt $c$ concentrations in the range from $5.0 \times 10^{-8}$ to $1.0 \times 10^{-4} \mathrm{M}$ with detection limit of $2.0 \times 10^{-8} \mathrm{M}$. Similarly, Wang et al., prepared and ECL nanocomposite probe by combing CdSE quantum dots, graphene oxide, and chitosan(Wang et al., 2012) and employed it for detecting cyt $c$.

\subsubsection{Resonance Rayleigh scattering}

Resonance Rayleigh scattering (RRS) is a special elastic scattering generated when the wavelength of Rayleigh scattering is located at the molecular absorption bondor close to it. Recently, novel materials, such as nanoparticles in association with the RRS technique, have been successfully utilized for analysis of proteins. For example, Ya et al., demonstrated an RRS assay of cyt $c$ using Glutathione (GSH) capped $\mathrm{Ag}_{2}$ Te nanoparticles (Yan et al., 2016). The RRS intensity of nanocomposite optical probe (negative charge) is enhanced greatly when positively charged cyt $c$ mixed. Real time production of cyt $c$ in living cells can be dynamically imaged using plasmon resonance energy transfer (PRET) spectroscopy.

\subsubsection{Label-free immunosensors}

Although labelled or mediator based immunosensors are widely used for protein detection, however, it was reported that the long term presence of the redox probe influences the activity of the immobilized antibody (Rickert et al., 1996). Label-free direct electrochemical immunosensor thus can be useful to detect proteins possessing metal prosthetic group with reversible redox activity. The direct label-free electrochemical immunosensors for metalloprotein are very attractive, since it requires simple assay procedure that does not require a complicated labeling process to measure the antigen. 


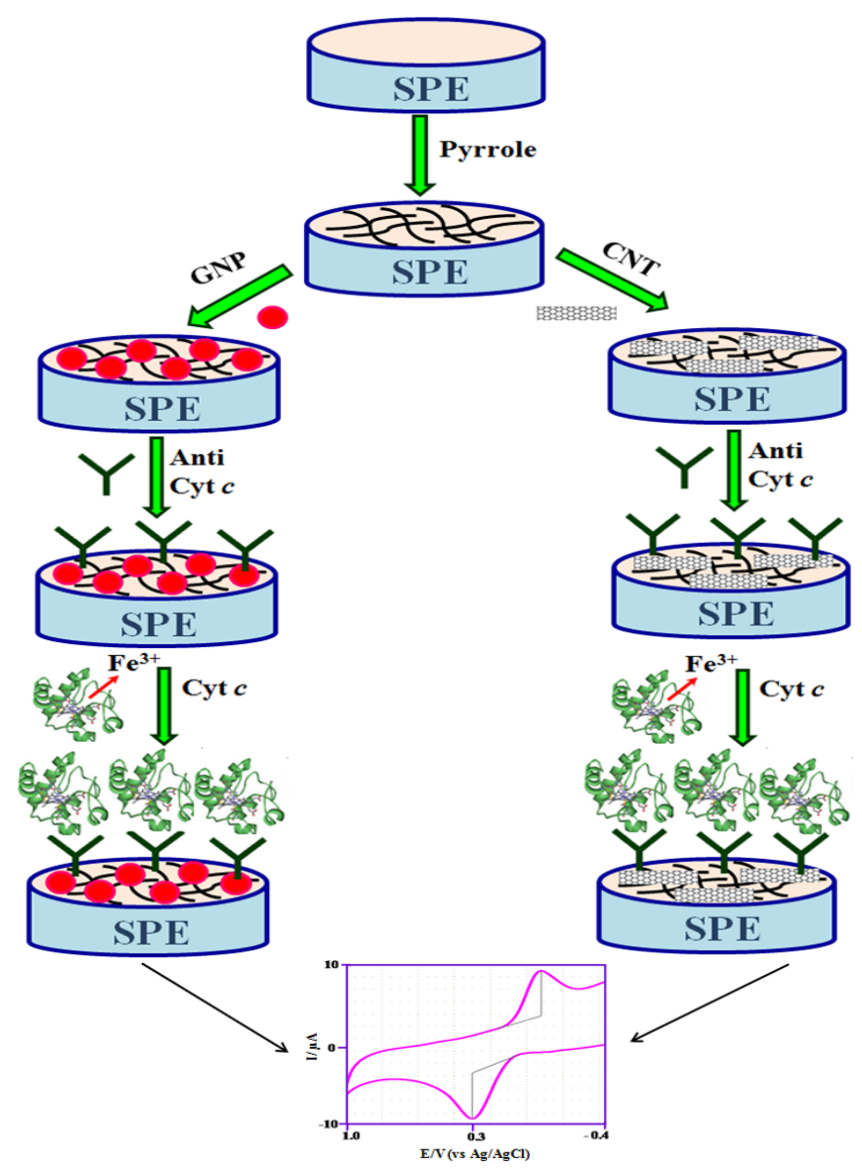

Figure 14. Schematic representation of step-wise fabrication of the label-free cyt $c$ immunosensors (Pandiaraj et al., 2014b) (reproduced with permission from Elsevier Limited)

For detection of cyt $c$, the specific cyt $c$ monoclonal anti-cyt $c$ were immobilized on the electrode to capture cyt $c$, and then the cyt $c$ adsorbed electrode measured through its electrochemical activity due to heme of cytc (Fe (III)/Fe (II)). This direct redox response was effectively utilized here for the quantitative measurement of cyt $c$ without usage of any redox probe or enzymatic label. Further, the direct electron transfer of cyt $c$ with the electrode surface was enhanced by modifying the electrode surfaces with nanostructures. Our group reported two types of nano-architectured immunosensor platforms to improve the direct electroactivity of cyt $c$ (Pandiaraj et al., 2014b). The first configuration involved the SAM modification on GNP in PPy tailored SPE. Second architecture features an effective incorporation of CNT using nafion on PPy modified SPE (Fig. 14). However, the overall analytical performance of GNP based immunosensors (detection limit $2 \mathrm{nM}$; linear range: $2 \mathrm{nM}-150 \mu \mathrm{M}$; sensitivity: $154 \mathrm{nA} \mathrm{nM}^{-1}$ ) was better than the CNT-PPy (detection limit $10 \mathrm{nM}$; linear range: $10 \mathrm{nM}-50 \mu \mathrm{M}$; sensitivity: 
$122 \mathrm{nA} \mathrm{nM}^{-1}$ ). The greater analytical performance of the anti-cyt $c / \mathrm{SAM} / \mathrm{GNP} / \mathrm{PPy}$ immunosensor is attributed to the densely and uniformly distributed GNP that greatly increased the active surface area for the formation of SAM of cysteine. The well-organized SAM on GNP/PPy nanocomposite also made it easier to form the stable and covalent immobilization of huge number of anti-cyt $c$ which could further improve the sensitivity and linear range of the immunosensor.

Wen et al., have developed an immunosensor for cyt $c$ by immobilizing anti-cyt $c$ at the interface of gold nanoparticle-polydopamine (AuNP/PDA) composites. This method achieved a linear range $(0.1-100 \mu \mathrm{M})$ for standard cyt $c$ with a detection limit of $0.03 \pm 0.01 \mu \mathrm{M}$. EIS and differential pulse voltammetry (DPV) were used to measure cyt $c$ concentrations. The anti-cyt $c$ or anti-caspase-9 functionalized-immunosensor provided a biomimetic interface for immunosensing of cyt $c$ or caspase-9 in Hela cells during apoptosis and the results were validated with flow cytometry analysis(Wen et al., 2014). Intracellular cyt $c$ measurement in a single cell using optical nanobiosensor was demonstrated by Song and coworkers. The optical nanobiosensor composed of a plastic-clad silica fibers with $600-\mu \mathrm{m}$ and immobilized anti-cyt $c$, labelled with biotin conjugate. Fluorescent measurement with the help of biotin conjugate solution was used to detect cyt $c$ release during photo induced apoptosis of cancer cells. The results of the labeled nanobiosensor was correlated with standard ELISA protocols (Joon Myong Song et al., 2004).

\subsection{Nucleic acid based cyt $c$ biosensors}

\subsubsection{DNA based cyt $c$ biosensors}

Since each organism has unique DNA sequences, any self-replicating microorganism can be easily identified. Biosensors based on nucleic acid as biorecognition element are simple, rapid, and inexpensive and hence it is widely used in protein detection. Besides enzymes and antibodies, nucleic acids (especially DNA) are also widely used to detect cyt $c$. The nonenzymatic DNA based cyt $c$ biosensor mainly uses the electrostatic interaction between the cyt $c$ and the DNA to detect cyt $c$. Cyt $c$ is a positively charged protein and has lysine residues surrounded by its heme prosthetic group.

A sensor for cyt $c$ was developed using a carbon paste electrode (CPE) modified with cellulose-DNA was reported by Lee et al. Since DNA has negative charged phosphate groups, 
which can electrostatically adsorbed with positively charged cyt $c$. The magnitude of the redox current observed for adsorbed cyt $c$ was directly proportional to the concentration of cyt $c$ present in the solution. The cellulose-DNA modified CPE results in a linear relationship at a concentration of cyt $c$ between $1 \mu \mathrm{M}$ to $100 \mu \mathrm{M}( \pm 5 \%$ at $n=5)$. The detection limit of cyt $c$ was estimated to be $0.5 \mu \mathrm{M}(\mathrm{S} / \mathrm{N}=3)$. A real sample analysis was carried out for cyt $c$ in a rat mitochondria fraction to evaluate the validation of this method (Lee et al., 2004). Similarly, Liu and coworkers reported a simple approach for detect cyt $c$ using DNA-modified glassy carbon electrode(Liu et al., 2006). The direct electron transfer of horse heart cyt $c$ on DNA-modified glassy carbon electrode was examined using differential pulse voltammetry for the detection of cyt $c$. In this case a linear relationship was obtained for the cyt $c$ concentration range from 4.0 $\times 10^{-6} \mathrm{M}$ to $1.2 \times 10^{-5} \mathrm{M}$ with a detection limit of $1.0 \times 10^{-6} \mathrm{M}(\mathrm{S} / \mathrm{N}=3)$

Later on, Liu and Wei described the detection of cyt $c$ at biocompatible gold nanoparticle supported bilayer lipid membrane (sBLM) modified with anionic sites(Liu and Wei, 2008). They have deposited $\mathrm{Au}$ nanoparticles electrochemically through the sBLM modified with lauric acid to build the anionic sites modified nanoscale electrode array to detect cyt $c$. The detection limit of this electrode was $50 \mathrm{nM}$ for cyt $c(\mathrm{~S} / \mathrm{N}=3)$ and the linear range of cyt $c$ concentration spans between $1.0 \mathrm{X} 10^{-7}$ and $3.2 \times 10^{-6} \mathrm{M}$. Similarly, Zhao et al., have also constructed a selfassembled gold nanoparticles multilayer architecture on a gold electrode based on the recognition between complementary oligo-DNA strands (Zhao et al., 2008). The negatively charged DNA is negatively charged, it is rational that cyt $c$ with positive charge in $\mathrm{pH} 7.4$ buffer can easily bind to HS-ssDNA modified on either the AuNPs or the substrate gold electrode surface via orientated electrostatic interaction. In some cases, researchers studied the direct electrochemistry of cyt $c$ at nanomaterial modified electrode for its detection. Wang et al., 2002 reported the detection of cyt $c$ using its direct electron transfer at CNT modified electrode (Jianxiu Wang et al., 2002). Similarly, the direct electron transfer between GNP and cyt $c$ for the cyt $c$ sensing has been reported by Wang et al., (Wang and Wang, 2004). These methods, however, suffer from lack of selectivity for the detection of cyt $c$, especially in tissue or biological samples, due to the fact that the interaction of the recognition elements (negative charge) with the cyt $c$ is only based on electrostatic interaction. Still, these methods are prone to interferences by other positively charged species present in the samples and hence are not applicable to the biological systems. 


\subsubsection{Aptamer based cyt $c$ biosensors}

Aptasensors are biosensors that use aptamers as the biorecognition element. Aptamers are artificial DNA or RNA oligonucleotides selected in vitro which have the ability to bind to proteins, small molecules or even whole cells, recognizing their target with high affinity and specificity similar to those of antibodies. Ocaña and coworkers reported a label-free aptasensor for the detection of cyt $c$. Graphite-epoxy composite (GEC) electrode was employed to immobilize the aptamers by simple wet physical adsorption procedure (Ocaña et al., 2014). Electrochemical impedance spectroscopy technique was used to monitor the biosensing event and confirmation of steps performed for building the cyt $c$ aptasensor using $\left[\mathrm{Fe}(\mathrm{CN})_{6}\right]^{3-/ 4-}$ redox marker. The aptasensor has a good detection range for cyt $c$ between $50 \mathrm{pM}$ and $50 \mathrm{nM}$, as well as a high sensitivity with a low detection limit of $63.2 \mathrm{pM}$.

Loo et al., have designed a new type of cyt $c$ aptasensor using bio-barcode (BBC) assay(Loo et al., 2014, 2013). They used an aptamer- based bio-barcode (ABC) to detect a cell death marker, cyt $c$ and its subsequent application to screen anti-cancer drugs. Normally, the $\mathrm{BBC}$ assay involves a magnetic microparticle (with antibody to capture the target of interest) and gold nanoparticle (with recognition antibody and thiolated single-stranded barcode DNAs) to form a sandwich around the target. The concentration of target is determined by the amount of barcode DNA released from the nanoparticles. Different from the conventional immunoassays such as ELISA using enzyme to amplify signals, the BBC assay amplifies signals by using PCR. Two types of particles are involved in the assay. The first is a magnetic bead conjugated with polyclonal capturing antibodies. The second is a gold nanoparticle coated with monoclonal recognition antibody for the same target so that an immuno-sandwich is formed with the magnetic bead. The second nanoparticle also carries hundreds of single-stranded DNA molecules of known sequence that hybridizes with its complementary DNA through Watson-Crick base pairings. These hybridized DNA are the barcode markers. After reaction with the analytes, sandwich structures are obtained by magnetic separation and marker DNA are released by melting and serve as templates in PCR amplifications. These marker DNA can be detected easily by standard techniques such as agarose electrophoresis or real-time PCR technique. The aptamer $\mathrm{BBC}$ assay is highly sensitive and the whole process can be finished only in $3 \mathrm{hrs}$. The efficacy of various drugs with potential anti-cancer activity was also assessed. 
In a work by Poturnayova ${ }^{a}$ et al., binding response of aptamers specific to cyt $c$ was analyzed by thickness shear mode acoustic methods. The results were compared with the electrochemical technique and found that the detection of cyt $c$ by electrochemical transducer was less favorable in comparison with acoustic transducer. Electro-chemiluminescence and fluorescence based cyt $c$ apta sensors has also been reported (vide supra) (Bin et al., 2016; Yin et al., 2015). Stepnova et al., proposed a novel electrochemical aptasensor for detecting cyt c on the base of glassy carbon electrode modified electropolymerized neutral red and decacarboxylated pillar[5] arene $(\mathrm{P}[5] \mathrm{A}-\mathrm{COOH})$ bearing terminal neutral red and aminated aptamer specific to cyt $c$ (Stepanova et al., 2016). Surface-enhanced Raman scattering (SERS) based aptasensor has also been reported for the detection of cyt (Xia et al., n.d.)c. The target binding event leads to an enhancement in SERS intensity of SERS-active molecule adsorbed on the gold surface. The detection limit of the sensor is $2 \mathrm{nM}$ with a dynamic range spanning to $1 \mu \mathrm{M}$.

Table 1: Comparison of analytical parameters of the cyt $\mathrm{c}$ biosensors

\begin{tabular}{lllll}
\hline \multicolumn{1}{c}{ Sensor architecture } & Technique & \multicolumn{1}{c}{ Linearity } & Detection limit & \multicolumn{1}{c}{ Reference } \\
\hline ssDNA/GNP/Au & $\mathrm{CV}$ & $2 \mathrm{nM}-0.1 \mu \mathrm{M}$ & $0.67 \mathrm{nM}$ & (Zhao et al., 2008) \\
sBLM/GNP/PC/LA/GCE & $\mathrm{LSV}$ & $0.1 \mu \mathrm{M}-3.2 \mu \mathrm{M}$ & $50 \mathrm{nM}$ & (Liu and Wei, 2008) \\
$\mathrm{CcO} / \mathrm{NiONPs} / \mathrm{MWCNT/PANI/Au}$ & $\mathrm{CV}$ & $5 \mathrm{pM}-0.5 \mu \mathrm{M}$ & $5 \mathrm{pM}$ & (Batra et al., 2013) \\
$\mathrm{CcO} / \mathrm{DDAB} / \mathrm{Au}$ & $\mathrm{SWV}$ & $0.2 \mu \mathrm{M}-800 \mu \mathrm{M}$ & $0.2 \mu \mathrm{M}$ & (Ashe et al., 2007) \\
$\mathrm{CcR} / \mathrm{SAM} / \mathrm{GNP} / \mathrm{PPy} / \mathrm{Pt}$ & $\mathrm{CV}$ & $5 \mu \mathrm{M}-600 \mu \mathrm{M}$ & $2 \mu \mathrm{M}$ & (Pandiaraj et al., 2013b) \\
$\mathrm{CcR} / \mathrm{CNT} / \mathrm{PPy} / \mathrm{Pt}$ & $\mathrm{CV}$ & $1 \mu \mathrm{M}-1000 \mu \mathrm{M}$ & $0.5 \mu \mathrm{M}$ & (Pandiaraj et al., 2013b) \\
$\mathrm{CcR} / \mathrm{CNT} / \mathrm{PPy} / \mathrm{SPE}$ & $\mathrm{CV}$ & $50 \mathrm{nM}-500 \mu \mathrm{M}$ & $50 \mathrm{nM}$ & (Pandiaraj et al., 2014a) \\
anti-cyt $c / \mathrm{SAM} / \mathrm{GNP} / \mathrm{PPy} / \mathrm{SPE}$ & $\mathrm{CV}$ & $2 \mathrm{nM}-150 \mu \mathrm{M}$ & $2 \mathrm{nM}$ & (Pandiaraj et al., 2014b) \\
anti-cyt $c / \mathrm{CNT} / \mathrm{PPy} / \mathrm{SPE}$ & $\mathrm{CV}$ & $10 \mathrm{nM}-50 \mu \mathrm{M}$ & $10 \mathrm{nM}$ & (Pandiaraj et al., 2014b) \\
PEG/Aptamer/Epoxy-Graphite & $\mathrm{EIS}$ & $50 \mathrm{pM}-50 \mathrm{nM}$ & $63.2 \mathrm{pM}$ & (Ocaña et al., 2014) \\
Aptamer/EDC-NHS/MUA/Gold & $\mathrm{SPR}$ & $80 \mathrm{nM}-80 \mathrm{pM}$ & $\geq 50 \mathrm{pM}$ & (Loo et al., 2014) \\
DNA-Cellulose/CPE & $\mathrm{DPV}$ & $0.1 \mathrm{mM}-1 \mu \mathrm{M}$ & $0.5 \mu \mathrm{M}$ & (Lee et al., 2004) \\
\hline
\end{tabular}

Single-strand DNA (ssDNA), Supported bilayer lipid membrane (sBLM), Soybean Phosphatidylcholine, (PC), Lauric acid (LA), Glassy carbon electrode (GCE), Nickel oxide nanoparticles (NiONPs), Polyaniline (PANI), didodecyldimethylammonium bromide (DDAB), Polyethylene glycol (PEG), 11-mercaptoundecanoic acid (MUA), Carbon paste electrode (CPE).

\section{Trend towards POC cyt $c$ measurement}


POC biosensors allows the detection of wide range of biomarkers at clinical settings. These systems provides the advantages of rapid results and thus ensuring earlier diagnosis and effective treatment. To use in clinical settings and POC diagnosis, the biosensor has to be small, produce rapid results, cost-effective, stable, and should offer continuous/frequent monitoring. The advances in microelectronics and an inherent simplicity afforded by the electrochemical transducers have allowed a novel way to fabricate miniaturized and low-cost biosensors for routine clinical diagnosis. Our group have recently reported, a portable, cost-effective electrochemical assay for rapid, sensitive, and quantitative detection of cyt $c$ release(Pandiaraj et al., 2014a). The fabricated device comprising CcR-CNT-PPy nanocomposite modified SPE integrated with a low-cost PIC microcontroller based data acquisition unit performing cyclic voltammetry for the measurement of cyt $c$ (Fig. 15). Further, this assay was successfully applied to measure cyt $c$ release from cardiomyocytes and the results were well correlated with standard ELISA. Since the oxidative stress can cause apoptosis to cardiomyocytes through an increased production of $\mathrm{H}_{2} \mathrm{O}_{2}$ in cells, we treated cardiomyocytes with $\mathrm{H}_{2} \mathrm{O}_{2}$ to mimic the cell death by oxidative stress. Cardiomyocytes cell lines were treated with $50 \mu \mathrm{M}$ and $200 \mu \mathrm{M} \mathrm{H}_{2} \mathrm{O}_{2}$ for $24 \mathrm{~h}$ to induce the cell death. Exposure of cultured cardiomyocytes to $\mathrm{H}_{2} \mathrm{O}_{2}$ led to a dose dependent decrease in cell viability, as assessed by MTT assay and miniaturized cyt c biosensor. In the future, the realization of commercial applications will hinge on the continuous improvements in the stability, reproducibility, sensitivity, and specificity of the devices in combination with industrial developments of low-cost processing techniques.

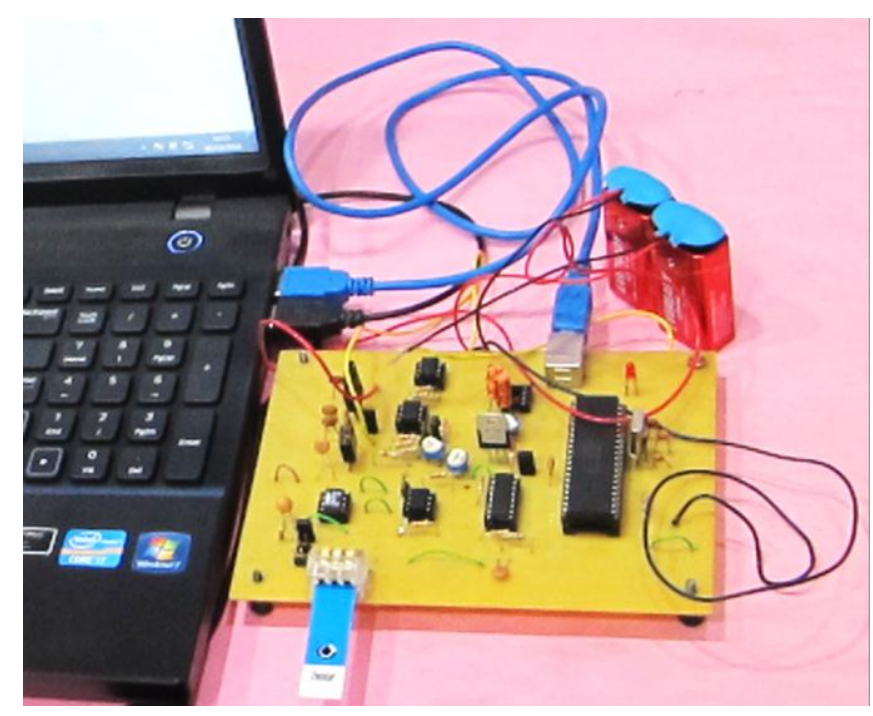


Figure 15. Photograph of the USB based electrochemical analyzer showing the interface with cyt $c$ biosensor and laptop(Pandiaraj et al., 2014a) (reproduced with permission from Elsevier Limited).

\section{Future perspective and conclusions}

This review compiles, for the first time, role of cyt $c$ in various biochemical and biomedical process and techniques employed for its detection. The release of cyt $c$ from mitochondria results in activation of cell death pathways and has been demonstrated to play an important role in various neurodegenerative disorders. Studies have that cyt $c$ can also act as an antioxidative enzyme in the mitochondria by removing ROS from mitochondria. The detection of cyt $c$ release is thus critically important as this not only provides valuable information about the nature and extent of apoptosis but serves as a preclinical indicator of various pathologies, medical diagnostics and therapeutic treatment. For example, detection of serum cyt $c$ levels during cancer diagnosis would provide valuable information about prognosis and suggests severity of the disease in cancer patients. However, monitoring of serum cyt $c$ during chemotherapy serve as a sensitive cell death marker to assess the efficacy of cancer therapy and screening of an appropriate anti-cancer drug. Early detection of serum cyt $c$ serves as a preclinical indicator of various pathologies including myocardial infarctions, brain damage, and severe liver damage. Thus early detection and continuous monitoring of cyt $c$ would allow an individual's more effective patient adjusted therapy with lesser side effects and good clinical outcomes. Researchers have designed novel biosensing technologies that could detect physiological concentration of cyt $c$. The recent advancements in biosensor technologies have enabled the transition of clinical laboratory cyt $c$ assay to near-patient settings, and provide therapist and biologist with timely diagnostic details to make appropriate decisions regarding diagnosis and treatment. Such developed next generation future sensors would be useful for cyt c related diseases diagnostics and in treatment decision. Enzymatic sensors (both $\mathrm{CcO}$ and $\mathrm{CcR}$ based biosensor) offers continuous monitoring of cyt $c$ release while antibody and aptamer based sensors would be suitable for designing a disposable biosensor chip, the one similar to commercial flow immunoassay chips. Successful application of smart nanomaterial to develop miniaturized sensor and further integration to developed portable sensing systems for cyt $c$ 
detection offers faster, selective, and higher sensitivity than standard ELISA techniques. The progress made in label-free immunosensing and enzymatic biosensing format for cyt $c$ detection enable continuous monitoring of cyt $c$ without the need for any additional reagents.

Finally, this review would serve as a call to promote research related to design of newer biosensing technologies based on various receptor and efficient transducer systems to detect cyt $c$. We believe that there is a considerable scope to design smart and efficient sensing systems for detecting cyt $c$ at POC application. Such developed POC device would be easy to handle, need low-power to operate, cost-effective, and performing detection in-field/on-site. Such developed system combined with clinical trials correlate cyt $c$ concentration with clinical abnormalities and may provide useful bio-informatics needed to decide therapeutics timely and diseases management for personalized health care.

\section{Acknowledgements:}

Authors acknowledge National Science Foundation (NSF), ASSIST Nanosystems ERC (EEC1160483).

\section{References:}

Ashe, D., Alleyne, T., Iwuoha, E., 2007. Serum cytochrome c detection using a cytochrome c oxidase biosensor. Biotechnol. Appl. Biochem. 46, 185-9. doi:10.1042/BA20060103

Barczyk, K., Kreuter, M., Pryjma, J., Booy, E.P., Maddika, S., Ghavami, S., Berdel, W.E., Roth, J., Los, M., 2005. Serum cytochrome c indicates in vivo apoptosis and can serve as a prognostic marker during cancer therapy. Int. J. cancer 116, 167-73. doi:10.1002/ijc.21037

Batra, B., Lata, S., Rani, S., Pundir, C.S., 2013. Fabrication of a cytochrome c biosensor based on cytochrome oxidase/NiO-NPs/cMWCNT/PANI modified Au electrode. J. Biomed. Nanotechnol. 9, 409-16.

Bin, N., Li, W., Yin, X., Huang, X., Cai, Q., 2016. Electrochemiluminescence aptasensor of TiO2/CdS:Mn hybrids for ultrasensitive detection of cytochrome c. Talanta 160, 570-576. doi:10.1016/j.talanta.2016.07.046

Brown, G.C., Borutaite, V., 2008. Regulation of apoptosis by the redox state of cytochrome c. 
Biochim. Biophys. Acta - Bioenerg. 1777, 877-881. doi:10.1016/j.bbabio.2008.03.024

Burdon, R.H., Gill, V., Rice-Evans, C., 1989. Cell proliferation and oxidative stress. Free Radic. Res. Commun. 7, 149-59.

Burgess, J.D., Rhoten, M.C., Hawkridge, F.M., 1998. Observation of the Resting and Pulsed States of Cytochrome c Oxidase in Electrode-Supported Lipid Bilayer Membranes. J. Am. Chem. Soc. 120, 4488-4491. doi:10.1021/ja974275z

Campos, C.B.L., Paim, B.A., Cosso, R.G., Castilho, R.F., Rottenberg, H., Vercesi, A.E., 2006. Method for monitoring of mitochondrial cytochrome $\mathrm{c}$ release during cell death: Immunodetection of cytochrome $\mathrm{c}$ by flow cytometry after selective permeabilization of the plasma membrane. Cytometry. A 69, 515-23. doi:10.1002/cyto.a.20273

Cao, M., Cao, C., Liu, M., Wang, P., Zhu, C., 2009. Selective fluorometry of cytochrome c using glutathione-capped CdTe quantum dots in weakly basic medium. Microchim. Acta 165, 341-346. doi:10.1007/s00604-009-0140-8

Carvajal, M.A., Ballesta-Claver, J., Morales, D.P., Palma, A.J., Valencia-Mirón, M.C., CapitánVallvey, L.F., 2012. Portable reconfigurable instrument for analytical determinations using disposable electrochemiluminescent screen-printed electrodes. Sensors Actuators B Chem. 169, 46-53. doi:10.1016/j.snb.2012.01.072

Chen, T.-T., Tian, X., Liu, C.-L., Ge, J., Chu, X., Li, Y., 2015. Fluorescence Activation Imaging of Cytochrome c Released from Mitochondria Using Aptameric Nanosensor. J. Am. Chem. Soc. 137, 982-989. doi:10.1021/ja511988w

Crofts, A.R., Holland, J.T., Victoria, D., Kolling, D.R.J., Dikanov, S.A., Gilbreth, R., Lhee, S., Kuras, R., Kuras, M.G., 2008. The Q-cycle reviewed: How well does a monomeric mechanism of the bc(1) complex account for the function of a dimeric complex? Biochim. Biophys. Acta 1777, 1001-19. doi:10.1016/j.bbabio.2008.04.037

Cullison, J.K., Hawkridge, F.M., Nakashima, N., Yoshikawa, S., 1994. A Study of Cytochrome c Oxidase in Lipid Bilayer Membranes on Electrode Surfaces. Langmuir 10, 877-882. doi:10.1021/la00015a044

Culmsee, C., Landshamer, S., 2006. Molecular insights into mechanisms of the cell death program: role in the progression of neurodegenerative disorders. Curr. Alzheimer Res. 3, $269-83$.

Dixit, C.K., Kumar, A., Kaushik, A., 2012. Nanosphere lithography-based platform for 
developing rapid and high sensitivity microarray systems, Biochemical and Biophysical Research Communications. doi:10.1016/j.bbrc.2012.05.144

Dong, Y.-P., Wang, J., Peng, Y., Zhu, J.-J., 2016. Electrogenerated chemiluminescence resonance energy transfer between luminol and CdS/graphene nanocomposites and its sensing application. J. Electroanal. Chem. doi:10.1016/j.jelechem.2016.05.024

Feissner, R., Xiang, Y., Kranz, R.G., 2003. Chemiluminescent-based methods to detect subpicomole levels of c-type cytochromes. Anal. Biochem. 315, 90-4.

Friedrich, M.G., Plum, M.A., Santonicola, M.G., Kirste, V.U., Knoll, W., Ludwig, B., Naumann, R.L.C., 2008. In situ monitoring of the catalytic activity of cytochrome C oxidase in a biomimetic architecture. Biophys. J. 95, 1500-10. doi:10.1529/biophysj.107.122747

Fuku, X., Iftikar, F., Hess, E., Iwuoha, E., Baker, P., 2012. Cytochrome c biosensor for determination of trace levels of cyanide and arsenic compounds. Anal. Chim. Acta 730, 4959. doi:10.1016/j.aca.2012.02.025

Gilbert, L., Jenkins, A.T.A., Browning, S., Hart, J.P., 2011. Development of an amperometric, screen-printed, single-enzyme phosphate ion biosensor and its application to the analysis of biomedical and environmental samples. Sensors Actuators B Chem. 160, 1322-1327. doi:10.1016/j.snb.2011.09.069

Goldstein, J.C., Muñoz-Pinedo, C., Ricci, J.-E., Adams, S.R., Kelekar, A., Schuler, M., Tsien, R.Y., Green, D.R., 2005. Cytochrome c is released in a single step during apoptosis. Cell Death Differ. 12, 453-62. doi:10.1038/sj.cdd.4401596

Green, D.R., Goldstein, J.C., Waterhouse, N.J., Juin, P., Evan, G.I., 2000. The coordinate release of cytochrome c during apoptosis is rapid,complete and kinetically invariant. Nat. Cell Biol. 2, 156-162. doi:10.1038/35004029

Guodong Liu, $\uparrow, \uparrow$, Ying-Ying Lin, $\uparrow, \S$, Jun Wang, $\uparrow$, Hong Wu, $\uparrow$, Chien M. Wai, $\S$. and, Yuehe Lin*, $\uparrow, 2007$. Disposable Electrochemical Immunosensor Diagnosis Device Based on Nanoparticle Probe and Immunochromatographic Strip.

Hatefi, Y., 1985. The mitochondrial electron transport and oxidative phosphorylation system. Annu. Rev. Biochem. 54, 1015-69. doi:10.1146/annurev.bi.54.070185.005055

Hayashi, H., Capaldi, R.A., 1972. The proteins of the outer membrane of beef heart mitochondria. Biochim. Biophys. Acta - Biomembr. 282, 166-173. doi:10.1016/00052736(72)90320-3 
Hrabakova, J., Ataka, K., Heberle, J., Hildebrandt, P., Murgida, D.H., 2006. Long distance electron transfer in cytochrome c oxidase immobilised on electrodes. A surface enhanced resonance Raman spectroscopic study. Phys. Chem. Chem. Phys. 8, 759-66. doi:10.1039/b513379n

Hu, X.-W., Mao, C.-J., Song, J.-M., Niu, H.-L., Zhang, S.-Y., Huang, H., 2013. Fabrication of $\mathrm{GO} / \mathrm{PANi} / \mathrm{CdSe}$ nanocomposites for sensitive electrochemiluminescence biosensor. Biosens. Bioelectron. 41, 372-378. doi:10.1016/j.bios.2012.08.054

Hüttemann, M., Pecina, P., Rainbolt, M., Sanderson, T.H., Kagan, V.E., Samavati, L., Doan, J.W., Lee, I., 2011. The multiple functions of cytochrome $\mathrm{c}$ and their regulation in life and death decisions of the mammalian cell: From respiration to apoptosis. Mitochondrion 11, 369-81. doi:10.1016/j.mito.2011.01.010

Javid, J., Mir, R., Julka, P.K., Ray, P.C., Saxena, A., 2015. Extracellular cytochrome c as a biomarker for monitoring therapeutic efficacy and prognosis of non-small cell lung cancer patients. Tumor Biol. 36, 4253-4260. doi:10.1007/s13277-015-3062-6

Jemmerson, R., LaPlante, B., Treeful, A., 2002. Release of intact, monomeric cytochrome c from apoptotic and necrotic cells. Cell Death Differ. 9, 538-548. doi:10.1038/sj.cdd.4400981

Jianxiu Wang, Meixian Li, Zujin Shi, Nanqiang Li, * and, Gu, Z., 2002. Direct Electrochemistry of Cytochrome c at a Glassy Carbon Electrode Modified with Single-Wall Carbon Nanotubes.

Joon Myong Song, Paul M. Kasili, Guy D. Griffin, and, Vo-Dinh*, T., 2004. Detection of Cytochrome c in a Single Cell Using an Optical Nanobiosensor.

Kadam, C.Y., Abhang, S.A., 2015. Serum levels of soluble Fas ligand, granzyme B and cytochrome c during adjuvant chemotherapy of breast cancer. Clin. Chim. Acta 438, 98102. doi:10.1016/j.cca.2014.08.012

Kadenbach, B., Hüttemann, M., 2015. The subunit composition and function of mammalian cytochrome c oxidase. Mitochondrion 24, 64-76. doi:10.1016/j.mito.2015.07.002

Kannan, Jain, 2000. Oxidative stress and apoptosis. Pathophysiology 7, 153-163.

Karunakaran, C., Madasamy, T., Pandiaraj, M., Sethy, N.K., Bhargava, K., 2014. Electrochemical Biosensors for Hypoxia Markers, in: Translational Research in Environmental and Occupational Stress. Springer India, New Delhi, pp. 93-107. doi:10.1007/978-81-322-1928-6_9 
Kavathia, N., Jain, A., Walston, J., Beamer, B.A., Fedarko, N.S., 2009. Serum markers of apoptosis decrease with age and cancer stage. Aging (Albany. NY). 1, 652-63. doi:10.18632/aging.100069

Kim, S.D., Moon, C.K., Eun, S.-Y., Ryu, P.D., Jo, S.A., 2005. Identification of ASK1, MKK4, JNK, c-Jun, and caspase-3 as a signaling cascade involved in cadmium-induced neuronal cell apoptosis. Biochem. Biophys. Res. Commun. 328, 326-34. doi:10.1016/j.bbrc.2004.11.173

King, M.A., Eddaoudi, A., Davies, D.C., 2007. A comparison of three flow cytometry methods for evaluating mitochondrial damage during staurosporine-induced apoptosis in Jurkat cells. Cytometry. A 71, 668-74. doi:10.1002/cyto.a.20428

Koopman, W.J.H., Verkaart, S., Visch, H.J., van Emst-de Vries, S., Nijtmans, L.G.J., Smeitink, J.A.M., Willems, P.H.G.M., 2007. Human NADH:ubiquinone oxidoreductase deficiency: radical changes in mitochondrial morphology? Am. J. Physiol. Cell Physiol. 293, C22-9. doi:10.1152/ajpcell.00194.2006

Kramer, D.M., Roberts, A.G., Muller, F., Cape, J., Bowman, M.K., 2004. Q-Cycle Bypass Reactions at the Qo Site of the Cytochrome bc1 (and Related) Complexes. Methods Enzymol. 382, 21-45. doi:10.1016/S0076-6879(04)82002-0

Kushnareva, Y., Murphy, A.N., Andreyev, A., 2002. Complex I-mediated reactive oxygen species generation: modulation by cytochrome $\mathrm{c}$ and $\mathrm{NAD}(\mathrm{P})+$ oxidation-reduction state. Biochem. J. 368, 545-53. doi:10.1042/BJ20021121

Langs-Barlow, A., Selvaraj, S., Ogbuagu, O., Shabanova, V., Shapiro, E.D., Paintsil, E., 2015. Association of circulating cytochrome $\mathrm{c}$ with clinical manifestations of antiretroviralinduced toxicity. Mitochondrion 20, 71-74. doi:10.1016/j.mito.2014.11.004

Lee, T.-Y., Kim, H.-J., Moon, J.-O., Shim, Y.-B., 2004. Determination of Cytochrome C with Cellulose- DNA Modified Carbon Paste Electrodes. Electroanalysis 16, 821-826. doi:10.1002/elan.200302885

Lewis, K.L., Su, L., Hawkridge, F.M., Ward, K.R., Rhoten, M.C., 2006. Immobilization of Cytochrome c Oxidase Into Electrode-Supported Lipid Bilayer Membranes for In Vitro Cytochrome c Sensing. IEEE Sens. J. 6, 420-427.

Li, J., Cheng, G., Dong, S., 1996. Direct electron transfer to cytochrome c oxidase in selfassembled monolayers on gold electrodes. J. Electroanal. Chem. 416, 97-104. 
doi:10.1016/S0022-0728(96)04732-8

Li, X., Liu, H., He, X., Song, Z., 2010. Determination of Cytochrome c in Human Serum and Pharmaceutical Injections Using Flow Injection Chemiluminescence. Appl. Biochem. Biotechnol. 160, 1065-1073. doi:10.1007/s12010-009-8598-8

Li, X.-M., Yang, X.-Y., Zhang, S.-S., 2008. Electrochemical enzyme immunoassay using model labels. TrAC Trends Anal. Chem. 27, 543-553. doi:10.1016/j.trac.2008.04.002

Lilja, H., Ulmert, D., Vickers, A.J., 2008. Prostate-specific antigen and prostate cancer: prediction, detection and monitoring. Nat. Rev. Cancer 8, 268-278. doi:10.1038/nrc2351

Liu, H., Sarnaik, S.M., Manole, M.D., Chen, Y., Shinde, S.N., Li, W., Rose, M., Alexander, H., Chen, J., Clark, R.S.B., Graham, S.H., Hickey, R.W., 2012. Increased cytochrome c in rat cerebrospinal fluid after cardiac arrest and its effects on hypoxic neuronal survival. Resuscitation 83, 1491-6. doi:10.1016/j.resuscitation.2012.04.009

Liu, Y., Wei, W., 2008. Detection of cytochrome c at biocompatible nanostructured Au-lipid bilayer-modified electrode. Anal. Sci. 24, 1431-6.

Liu, Y.-C., Cui, S.-Q., Yang, Z.-S., 2006. Direct electrochemical behavior of cytochrome c on DNA-modified glassy carbon electrode and its application to cytochrome c biosensor. Anal. Sci. 22, 1071-4.

Loo, F.-C., Ng, S.-P., Wu, C.-M.L., Kong, S.K., 2014. An aptasensor using DNA aptamer and white light common-path SPR spectral interferometry to detect cytochrome-c for anticancer drug screening. Sensors Actuators B Chem. 198, 416-423. doi:10.1016/j.snb.2014.03.077

Loo, J.F.C., Lau, P.M., Ho, H.P., Kong, S.K., 2013. An aptamer-based bio-barcode assay with isothermal recombinase polymerase amplification for cytochrome-c detection and anticancer drug screening. Talanta 115, 159-165. doi:10.1016/j.talanta.2013.04.051

Martinou, J.C., Desagher, S., Antonsson, B., 2000. Cytochrome c release from mitochondria: all or nothing. Nat. Cell Biol. doi:10.1038/35004069

Mathews, F.S., 1985. The structure, function and evolution of cytochromes. Prog. Biophys. Mol. Biol. 45, 1-56.

Michel, H., n.d. Cytochrome c Oxidase: Catalytic Cycle and Mechanisms of Proton Pumping-A Discussion. doi:10.1021/bi9910934

Millett, F., Havens, J., Rajagukguk, S., Durham, B.,. Design and use of photoactive ruthenium 
complexes to study electron transfer within cytochrome bc1 and from cytochrome bc1 to cytochrome c. Biochim. Biophys. Acta 1827, 1309-19. doi:10.1016/j.bbabio.2012.09.002

Ng, H., Smith, D.J., Nagley, P., 2012. Application of flow cytometry to determine differential redistribution of cytochrome $\mathrm{c}$ and Smac/DIABLO from mitochondria during cell death signaling. PLoS One 7, e42298. doi:10.1371/journal.pone.0042298

Ocaña, C., Arcay, E., del Valle, M., 2014. Label-free impedimetric aptasensor based on epoxygraphite electrode for the recognition of cytochrome c. Sensors Actuators B Chem. 191, 860-865. doi:10.1016/j.snb.2013.10.040

Pandiaraj, M., Benjamin, A.R., Madasamy, T., Vairamani, K., Arya, A., Sethy, N.K., Bhargava, K., Karunakaran, C., 2014a. A cost-effective volume miniaturized and microcontroller based cytochrome c assay. Sensors Actuators A Phys. 220, 290-297. doi:10.1016/j.sna.2014.10.018

Pandiaraj, M., Madasamy, T., Gollavilli, P.N., Balamurugan, M., Kotamraju, S., Rao, V.K., Bhargava, K., Karunakaran, C., 2013a. Nanomaterial-based electrochemical biosensors for cytochrome c using cytochrome c reductase. Bioelectrochemistry 91, 1-7. doi:10.1016/j.bioelechem.2012.09.004

Pandiaraj, M., Madasamy, T., Gollavilli, P.N., Balamurugan, M., Kotamraju, S., Rao, V.K., Bhargava, K., Karunakaran, C., 2013b. Nanomaterial-based electrochemical biosensors for cytochrome c using cytochrome c reductase. Bioelectrochemistry 91, 1-7. doi:10.1016/j.bioelechem.2012.09.004

Pandiaraj, M., Sethy, N.K., Bhargava, K., Kameswararao, V., Karunakaran, C., 2014b.

Designing label-free electrochemical immunosensors for cytochrome c using nanocomposites functionalized screen printed electrodes. Biosens. Bioelectron. 54, 115-21. doi:10.1016/j.bios.2013.10.030

Pasdois, P., Parker, J.E., Griffiths, E.J., Halestrap, A.P., 2011. The role of oxidized cytochrome c in regulating mitochondrial reactive oxygen species production and its perturbation in ischaemia. Biochem. J. 436, 493-505. doi:10.1042/BJ20101957

Patel, T., Gores, G.J., Kaufmann, S.H., 1996. The role of proteases during apoptosis. FASEB J. 10, 587-97.

Rhoten, M.C., Burgess, J.D., Hawkridge, F.M., 2002. The reaction of cytochrome c from different species with cytochrome c oxidase immobilized in an electrode supported lipid 
bilayer membrane. J. Electroanal. Chem. 534, 143-150. doi:10.1016/S0022-

0728(02)01138-5

Rhoten, M.C., Burgess, J.D., Hawkridge, F.M., 2000. Temperature and pH effects on cytochrome c oxidase immobilized in an electrode-supported lipid bilayer membrane. Electrochim. Acta 45, 2855-2860. doi:10.1016/S0013-4686(00)00359-5

Rickert, J., G6pel, W., Beck, W., Jung, G., Heiduschka, P., 1996. A \&quot;mixed' selfassembled monolayer for an impedimetric immunosensor. Biosens. Bioelectron. 11, 75768.

Rieger, M., Cervino, C., Sauceda, J.C., Niessner, R., Knopp, D., 2009. Efficient Hybridoma Screening Technique Using Capture Antibody Based Microarrays.

Sakaida, I., Kimura, T., Yamasaki, T., Fukumoto, Y., Watanabe, K., Aoyama, M., Okita, K., 2005. Cytochrome $\mathrm{c}$ is a possible new marker for fulminant hepatitis in humans. $\mathbf{J}$ Gastroenterol 40, 179-185. doi:10.1007/s00535-004-1517-4

Salamon, Z., Tollin, G., 1996. Surface plasmon resonance studies of complex formation between cytochrome $\mathrm{c}$ and bovine cytochrome $\mathrm{c}$ oxidase incorporated into a supported planar lipid bilayer. I. Binding of cytochrome $\mathrm{c}$ to cardiolipin/phosphatidylcholine membranes in the absence of oxida. Biophys. J. 71, 848-57. doi:10.1016/S0006-3495(96)79286-X

Shamsipur, M., Molaabasi, F., Hosseinkhani, S., Rahmati, F., 2016. Detection of Early Stage Apoptotic Cells Based on Label-Free Cytochrome c Assay Using Bioconjugated Metal Nanoclusters as Fluorescent Probes. Anal. Chem. 88, 2188-2197. doi:10.1021/acs.analchem.5b03824

Skemiene, K., Rakauskaite, G., Trumbeckaite, S., Liobikas, J., Brown, G.C., Borutaite, V., 2013. Anthocyanins block ischemia-induced apoptosis in the perfused heart and support mitochondrial respiration potentially by reducing cytosolic cytochrome c. Int. J. Biochem. Cell Biol. 45, 23-29. doi:10.1016/j.biocel.2012.07.022

Skulachev, V.P., 1998. Cytochrome $\mathrm{c}$ in the apoptotic and antioxidant cascades. FEBS Lett. 423, 275-80.

Stepanova, V.B., Shurpik, D.N., Evtugyn, V.G., Stoikov, I.I., Evtugyn, G.A., Osin, Y.N., Hianik, T., 2016. Label-free electrochemical aptasensor for cytochrome c detection using pillar[5]arene bearing neutral red. Sensors Actuators B 225, 57-65.

doi:10.1016/j.snb.2015.11.023 
Tait, S.W.G., Green, D.R., 2010. Mitochondria and cell death: outer membrane permeabilization and beyond. Nat. Rev. Mol. Cell Biol. 11, 621-32. doi:10.1038/nrm2952

Taufika Islam Williams, $\uparrow$, Kristina L. Toups, $\uparrow$, Diana A. Saggese, $\uparrow$, Kimberly R. Kalli, $\ddagger$, William A. Cliby, $\$$ and, David C. Muddiman*, $\uparrow, 2007$. Epithelial Ovarian Cancer:

Disease Etiology, Treatment, Detection, and Investigational Gene, Metabolite, and Protein Biomarkers.

Tsukihara, T., Aoyama, H., Yamashita, E., Tomizaki, T., Yamaguchi, H., Shinzawa-Itoh, K., Nakashima, R., Yaono, R., Yoshikawa, S., 1995. Structures of metal sites of oxidized bovine heart cytochrome c oxidase at 2.8 A. Science (80-. ). 269, 1069-1074. doi:10.1126/science. 7652554

Uttara, B., Singh, A. V, Zamboni, P., Mahajan, R.T., 2009. Oxidative stress and neurodegenerative diseases: a review of upstream and downstream antioxidant therapeutic options. Curr. Neuropharmacol. 7, 65-74. doi:10.2174/157015909787602823

Viswanathan, S., Rani, C., Vijay Anand, A., Ho, J.A., 2009. Disposable electrochemical immunosensor for carcinoembryonic antigen using ferrocene liposomes and MWCNT screen-printed electrode. Biosens. Bioelectron. 24, 1984-1989. doi:10.1016/j.bios.2008.10.006

Walker, A.M., Stevens, J.J., Ndebele, K., Tchounwou, P.B., 2016. Evaluation of Arsenic Trioxide Potential for Lung Cancer Treatment: Assessment of Apoptotic Mechanisms and Oxidative Damage. J. Cancer Sci. Ther. 8, 1-9. doi:10.4172/1948-5956.1000379

Wang, J., Li, M., Shi, Z., Li, N., Gu, Z., 2002. Direct Electrochemistry of Cytochrome c at a Glassy Carbon Electrode Modified with Single-Wall Carbon Nanotubes. Anal. Chem. 74, 1993-1997. doi:10.1021/ac010978u

Wang, L., Wang, E., 2004. Direct electron transfer between cytochrome c and a gold nanoparticles modified electrode, Electrochemistry Communications. doi:10.1016/j.elecom.2003.10.004

Wang, T., Zhang, S., Mao, C., Song, J., Niu, H., Jin, B., Tian, Y., 2012. Enhanced electrochemiluminescence of CdSe quantum dots composited with graphene oxide and chitosan for sensitive sensor. Biosens. Bioelectron. 31, 369-375. doi:10.1016/j.bios.2011.10.048

Wang, X., 2001. The expanding role of mitochondria in apoptosis. Genes Dev. 15, 2922-33. 
Wang, Y., Liu, J., Ma, A., Chen, Y., 2015. Cardioprotective effect of berberine against myocardial ischemia/reperfusion injury via attenuating mitochondrial dysfunction and apoptosis. Int. J. Clin. Exp. Med. 8, 14513-9.

Waterhouse, N.J., Trapani, J.A., 2003a. A new quantitative assay for cytochrome c release in apoptotic cells. Cell Death Differ. 10, 853-5. doi:10.1038/sj.cdd.4401263

Waterhouse, N.J., Trapani, J.A., 2003b. A new quantitative assay for cytochrome c release in apoptotic cells. Cell Death Differ. 10, 853-855. doi:10.1038/sj.cdd.4401263

Wen, Q., Zhang, X., Cai, J., Yang, P.-H., 2014. A novel strategy for real-time and in situ detection of cytochrome c and caspase-9 in Hela cells during apoptosis. Analyst 139, 2499506. doi:10.1039/c3an02205f

Xia, Y., Gao, P., Qiu, X., Xu, Q., Gan, S., Yang, H., Huang, S., n.d. Aptasensor based on triplex switch for SERS detection of cytochrome c. doi:10.1039/c2an36173f

Yan, S., Deng, D., Li, L., Chen, Y., Song, H., Lv, Y., 2016. Glutathione modified Ag2Te nanoparticles as a resonance Rayleigh scattering sensor for highly sensitive and selective determination of cytochrome C. Sensors Actuators B Chem. 228, 458-464. doi:10.1016/j.snb.2016.01.069

Yin, X., Cai, J., Feng, H., Wu, Z., Zou, J., Cai, Q., 2015. A novel VS 2 nanosheet-based biosensor for rapid fluorescence detection of cytochrome c. New J. Chem. 39, 1892-1898. doi:10.1039/C4NJ01971G

Yivgi-Ohana, N., Eifer, M., Addadi, Y., Neeman, M., Gross, A., 2011. Utilizing mitochondrial events as biomarkers for imaging apoptosis. Cell Death Dis. 2, e166. doi:10.1038/cddis.2011.47

Zhao, J., Zhu, X., Li, T., Li, G., 2008. Self-assembled multilayer of gold nanoparticles for amplified electrochemical detection of cytochrome c. Analyst 133, 1242-5. doi:10.1039/b806588h 